\title{
STOCHASTIC REACTION-DIFFUSION SYSTEMS WITH HÖLDER CONTINUOUS MULTIPLICATIVE NOISE
}

\author{
MARKUS C. KUNZE
}

\begin{abstract}
We prove pathwise uniqueness and strong existence of solutions for stochastic reaction-diffusion systems with locally Lipschitz continuous reaction term of polynomial growth and Hölder continuous multiplicative noise. Under additional assumptions on the coefficients, we also prove positivity of the solutions.
\end{abstract}

\section{INTRODUCTION}

Reaction-diffusion systems and stochastic perturbations of them play an important role in applications in chemistry, biology and physics [19. In an abstract form, a stochastic reaction-diffusion system can be treated as a stochastic evolution equation

$$
d U(t)=[A U(t)+F(U(t))] d t+G(U(t)) d W_{H}(t)
$$

on a Banach space $E$, which is a space of $\mathbb{R}^{r}$-valued functions, defined on a domain $\mathscr{O}$. Thus, (1.1) actually represents a system of $r$ coupled equations. Here, $A$ is a diagonal matrix of second order differential operators which describe the diffusion in the system and the map $F$ accounts for the reaction in the system and is typically a composition operator with components of polynomial growth. The system is driven by a cylindrical Wiener process $W_{H}$ in a suitable Hilbert space $H$.

In the case of $r=1$, i.e. a single reaction-diffusion equation rather than a system, there are many articles concerned with such equations, both in the case of additive noise (see [7, 11, 12, 8]) and in the case of (locally) Lipschitz continuous multiplicative noise (see 2, 4, 16, 27, 14]). Stochastic reaction-diffusion systems with locally Lipschitz continuous multiplicative noise were considered in [5].

In the case where the noise term $G$ is no longer locally Lipschitz continuous the techniques from the above references can no longer be used. This is essentially due to the fact that for such equations a priori only stochastically weak solutions (or martingale solutions) can be constructed. However, stochastic reaction-diffusion equations with merely Hölder continuous multiplicative noise appear naturally, e.g. in scaling limits of interacting particle systems, see e.g. [18] were for $r=1$ noise terms with $G(u)(x):=|u(x)|^{\frac{1}{2}}$ and $G(u)(x)=|u(x)(1-u(x))|^{\frac{1}{2}} \mathbb{1}_{\{0 \leq u(x) \leq 1\}}$ appear.

For such stochastic reaction-diffusion systems with Hölder continuous multiplicative noise only few results are available and, to the best of our knowledge, only the case $r=1$ is treated. In [3], existence of solutions has been proved under an additional boundedness assumption on the coefficient $G$. However, a uniqueness result is missing in that article, except for the case of locally Lipschitz continuous $G$. Pathwise uniqueness for the stochastic heat equation on $\mathbb{R}^{d}$ (i.e. $r=1, A$ is the Laplace operator and $F \equiv 0$ ) was proved in 21 in the case where $W_{H}$ is replaced with a colored noise and $G$ is composition with a $\gamma$-Hölder continuous function, where the

2010 Mathematics Subject Classification. 60H15, 35R60.

Key words and phrases. stochastic reaction diffusion system, pathwise uniqueness.

The author was supported by the Deutsche Forschungsgesellschaft in the framework of the DFG research training group 1100. 
allowed values of $\gamma$ depend on the noise. In [20, the one-dimensional version of the stochastic heat equation with white noise was considered, and it was proved that for that equation pathwise uniqueness holds for $\gamma>3 / 4$. For $\gamma<3 / 4$, it was recently proved in [17] that solutions are neither pathwise unique nor unique in law.

In this article, we are concerned with stochastic reaction-diffusion systems on a bounded Lipschitz domain $\mathscr{O} \subset \mathbb{R}^{d}$ of the form

$$
\left\{\begin{aligned}
d u_{l}(t, x)= & {\left[\mathscr{A}_{l} u_{l}(t, x)+f_{l}\left(x, u_{1}(t, x), \ldots, u_{r}(t, x)\right)\right] d t } \\
& \quad+\sum_{k=1}^{\infty} g_{l, k}\left(x, u_{l}(t, x)\right) d \beta_{l, k}(t), \\
& \quad t>0, x \in \mathscr{O}, l=1, \ldots, r \\
\frac{\partial u_{l}}{\partial \nu_{\mathscr{A}_{l}}}(t, x)= & 0 \quad t>0, x \in \partial \mathscr{O}, l=1, \ldots, r \\
u_{l}(0, x)= & \xi_{l}(x) \quad x \in \overline{\mathscr{O}}, l=1, \ldots, r .
\end{aligned}\right.
$$

Here, $\mathscr{A}_{l}$ is a uniformly elliptic, second order differential operator in divergence form on $\mathscr{O}$ and $\partial / \partial \nu_{A_{l}}$ is the associated conormal derivative. The functions $f_{l}$ : $\mathscr{O} \times \mathbb{R}^{k} \rightarrow \mathbb{R}$ are locally Lipschitz continuous, of polynomial growth and satisfy suitable dissipativity assumptions. Typical examples that we have in mind are odddegree polynomials with negative leading coefficients, see Section 6. The functions $g_{l, k}: \mathscr{O} \times \mathbb{R} \rightarrow \mathbb{R}$ are locally $\frac{1}{2}$-Hölder continuous and of linear growth such that the Hölder constants and the coefficients in the linear growth are square summable. Finally, the $\beta_{l, k}$ are independent, one-dimensional Brownian motions. By [17], pathwise uniqueness cannot be expected for equations driven by space-time white noise with $\frac{1}{2}$-Hölder continuous coefficient. However, our assumption allow us to consider noise terms of the form $G(U(t)) R d W$, where $G$ is composition with a locally $\frac{1}{2}$-Hölder continuous function of linear growth , $R$ is a Hilbert-Schmidt operator on $L^{2}(\mathscr{O})$ satisfying additional assumptions and $W_{H}$ is space-time white noise, see Example 2.4

We will make our assumptions precise in Section 2

Under these assumptions, we prove pathwise uniqueness (Theorem 3.1) of solutions to equation (1.2) on the state space $E=C(\overline{\mathscr{O}})^{r}$. Our proof follows the ideas of Yamada and Watanabe 29, who proved pathwise uniqueness for finite-dimensional SDE with $\frac{1}{2}$-Hölder continuous coefficients. The main difficulty to extend these results to the infinite-dimensional setting is of course to handle the differential operators $\mathscr{A}_{l}$. The strategy from [20, 21] to prove pathwise uniqueness, which is also an adaption of the Yamada-Watanabe ideas, cannot be used in our situation. Indeed, there the authors convolute solutions $u$ of the stochastic heat equation with a mollifier $\varphi_{n}$. In their variational framework, this yields the term $u * \Delta \varphi_{n}$ in the equation for the resulting process. It is then used that, as a consequence of its translation invariance, the Laplacian commutes with convolutions, i..e. we have $u * \Delta \varphi_{n}=\Delta\left(u * \varphi_{n}\right)$. This is no longer true for differential operators with nonconstant coefficients, which we consider here. We overcome this difficulty by using the concept of an (analytically) weak solution, see Definition 2.7, which allows us to perform pointwise estimates.

We would like to point out that in the Yamada-Watanabe result, it is essential that the multiplicative noise is diagonal. Therefore, in (1.2) we have also considered "diagonal noise" by letting the noise term in the $l$-th equation only depend only on $u_{l}$, rather than the whole vector $u$. Thus, the equations in (1.2) are only coupled via the reaction terms $f_{l}$

Under additional assumptions on the functions $f_{l}$ and $g_{l, k}$, similar arguments as in the proof of Theorem 3.1 can be used to show that the solutions to (1.2) preserve positivity, i.e. if $\xi_{1}, \ldots, \xi_{n} \geq 0$ a.s. then we also have $u_{l}(t) \geq 0$ almost surely for all $t \geq 0$ and $l=1, \ldots, r$, see Theorem 4.3 . 
Using pathwise uniqueness, we can adopt the strategy from [5, 14] to prove existence of solutions to (1.2) in Theorem 5.5. In contrast to the existence result from [3], we can drop the uniform boundedness assumption on $G$ and allow $G$ of linear growth.

This article is organized as follows. In Section 2, we fix our assumptions on equation (1.2) and rewrite it in the abstract form (1.1). We also prove some preliminary results and recall some stochastic concepts that we will use. Section 3 contains the prove of pathwise uniqueness and Section 4 our result about positivity of solutions. Existence of solutions will be proved in Section 5. In the concluding Section 6 . we apply our results to a stochastic reaction-diffusion system of Fitzhugh-Nagumo type.

\section{Preliminaries}

In this section, we fix our assumptions on equation (1.2), rewrite the equation in the abstract form (1.1) and recall different notions of existence and uniqueness for equation (1.1) that will be used in what follows.

Throughout, all vector spaces are real. If $H$ is a Hilbert space, we write $(\cdot, \cdot)_{H}$ for the inner product in $H$. When $P_{n}(u)$ and $Q_{n}(u)$ are certain quantities depending on an index $n$ and a function $u$, we write $P_{n}(u) \lesssim Q_{n}(u)$ to indicate that there exists a constant $c$, independent of $n$ and $u$ such that $P_{n}(u) \leq c Q_{n}(u)$ for all $n$ and $u$. We write $P_{n}(u) \approx Q_{n}(u)$ if both $P_{n}(u) \lesssim Q_{n}(u)$ and $Q_{n}(u) \lesssim P_{n}(u)$.

2.1. The differential operators. We assume that the differential operators $\mathscr{A}_{l}$ are given by

$$
\mathscr{A}_{l}=\sum_{i, j=1}^{d} D_{i}\left(a_{i j}^{l} D_{j}\right)-c_{l}
$$

where we make the following assumptions:

(A) The domain $\mathscr{O} \subset \mathbb{R}^{d}$ is bounded and has Lipschitz boundary. For $l=$ $1, \cdots, r$, the matrix valued functions $a_{l}:=\left(a_{i j}^{l}\right): \overline{\mathscr{O}} \rightarrow \mathbb{R}^{d \times d}$ are symmetric and have measurable entries. Moreover, for certain $\eta, M>0$ we have

$$
\eta|\xi|^{2} \leq \sum_{i, j=1}^{d} a_{i, j}(x) \xi_{i} \xi_{j} \leq M|\xi|^{2}
$$

for almost all $x \in \mathscr{O}$ and all $\xi \in \mathbb{R}^{d}$. Finally, $c_{l} \in L^{\infty}(\mathscr{O})$.

To construct realizations of the differential operators which are generators of strongly continuous semigroups, we follow a variational approach. We consider on $L^{2}(\mathscr{O})$ the symmetric form

$$
\mathfrak{a}^{l}[u, v]:=\int_{\mathscr{O}} \sum_{i, j=1}^{d} a_{i, j}^{l}(x) D_{i} u(x) \overline{D_{j} u(x)} d x+\int_{\mathscr{O}} u(x) v(x) c_{l}(x) d x
$$

endowed with domain $\mathrm{D}\left(\mathfrak{a}^{l}\right):=H^{1}(\mathscr{O})$. The associated operator $A_{l, 2}$ is given by

$$
\mathrm{D}\left(A_{l, 2}\right):=\left\{u \in H^{1}(\mathscr{O}): \exists w \in L^{2}(\mathscr{O}) \text { s.t. }(w, v)_{L^{2}}=\mathfrak{a}^{l}[u, v] \text { for all } v \in H^{2}(\mathscr{O})\right\}
$$

and $A_{l, 2} u=-w$. Note that the boundary condition $\partial u / \partial \nu_{\mathscr{A}_{l}}:=a_{l} \nabla u \cdot \nu=0$ on $\partial \mathscr{O}$ is incorporated in the domain of $A_{l, 2}$. As is well-known, the operator $A_{l, 2}$ generates a strongly continuous and analytic semigroup $S_{l, 2}$, see 26. Changing the function $c_{l}$ by a constant if necessary, we may and shall assume that $S_{l, 2}$ is uniformly exponentially stable, hence $A_{l, 2}$ is invertible. Note that we can compensate the change in $c_{l}$ by appropriately changing $f_{l}$ in equation (1.2), thus this assumption means no loss of generality for the stochastic reaction-diffusion equation (1.2).

Since the form $\mathfrak{a}$ is sub-Markovian, see [26. Chapter 4], the semigroup $S_{l, 2}$ restricts to a strongly continuous and analytic semigroup $S_{l, p}$ on $L^{p}(\mathscr{O})$ for every 
$p \in[2, \infty)$. The generator $A_{l, p}$ of the restricted semigroup $S_{l, p}$ is exactly the part of $A_{l, 2}$ in $L^{p}(\mathscr{O})$.

Finally, $\mathrm{D}\left(A_{l, 2}\right) \subset C(\overline{\mathscr{O}})$ and the semigroup $S_{l, 2}$ restricts to a strongly continuous semigroup $S_{l, C}$ on $C(\overline{\mathscr{O}})$, whose generator $A_{l, C}$ is the part of $A_{l, 2}$ in $C(\overline{\mathscr{O}})$. In the case where $c_{l} \equiv 0$, this follows from the results of [10], the case of general $c_{l} \in L^{\infty}(\mathscr{O})$ follows with a perturbation argument.

To prove existence of solutions to (1.2) in spaces of continuous functions, we will need some embedding results for the fractional domain spaces $\mathrm{D}\left(\left(-A_{l, p}\right)^{\alpha}\right)$. Often, such results are obtained from precise knowledge of $\mathrm{D}\left(A_{l, p}\right)$ and interpolation. However, this strategy requires more regularity of the coefficients $a_{l}$ and the boundary of $\mathscr{O}$. Here, we follow a different approach.

Lemma 2.1. Assume $(A)$ and let $d<2 p$. Then for $\alpha \in\left(\frac{d}{2 p}, 1\right)$, the fractional domain space $\mathrm{D}\left(\left(-A_{l, p}\right)^{\alpha}\right)$ is continuously embedded into $C(\overline{\mathscr{O}})$.

Proof. Let us first note that since $H^{1}(\mathscr{O}) \hookrightarrow L^{\frac{2 d}{d-2}}(\mathscr{O})$ for $d \geq 3$ (and $H^{1}(\mathscr{O}) \hookrightarrow$ $L^{q}(\mathscr{O})$ for arbitrary $q \in(1, \infty)$ for $\left.d \leq 2\right)$, it follows from [1, Section 7.3] that the semigroups $S_{l, p}$ are ultracontractive, i.e. for $p \geq 2$ the operator $S_{l, p}(t)$ maps $L^{p}(\mathscr{O})$ into $L^{\infty}(\mathscr{O})$ and we have

$$
\left\|S_{l, p}(t)\right\|_{\mathscr{L}\left(L^{p}(\mathscr{O}), L^{\infty}(\mathscr{O})\right)} \leq c t^{-\frac{d}{2 p}} \quad \forall 0<t \leq 1
$$

for a certain constant $c=c(l, p)>0$.

The fractional domain space $\mathrm{D}\left(\left(-A_{l, p}\right)^{\alpha}\right)$ is the range of the (bounded) operator $\left(-A_{l, p}\right)^{-\alpha}$, defined by

$$
\left(-A_{l, p}\right)^{-\alpha} f:=\frac{1}{\Gamma(\alpha)} \int_{0}^{\infty} t^{\alpha-1} S_{l, p}(t) f d t
$$

endowed with the norm $\left\|\left(-A_{l, p}\right) f\right\|_{\mathrm{D}\left(\left(-A_{l, p}\right)^{\alpha}\right)}:=\|f\|_{p}$. We note that since $S_{l, p}$ is analytic, $S_{l, p}(t) f \in \mathrm{D}\left(A_{l, p}\right)$ for all $t>0$ and $f \in L^{p}(\mathscr{O})$. As a consequence of [10], $\mathrm{D}\left(A_{l, p}\right) \subset C(\overline{\mathscr{O}})$, hence the integrand takes values in $C(\overline{\mathscr{O}})$.

Thus, to prove that $\left(-A_{l, p}\right)^{-\alpha} f \in C(\overline{\mathscr{O}})$, it suffices to show that the integral in (2.1) exists as a Bochner integral in $C(\overline{\mathscr{O}})$. Hence we have to show that $\left\|t^{\alpha-1} S_{l, p}(t) f\right\|_{\infty}$ is integrable on $(0, \infty)$. From ultracontractivity we obtain

$$
\left\|t^{\alpha-1} S_{l, p}(t) f\right\|_{\infty} \leq c t^{\alpha-1-\frac{d}{2 p}}\|f\|_{p} \quad \forall 0<t \leq 1
$$

which is integrable on $(0,1)$ since $\alpha>\frac{d}{2 p}$. Since $S_{l, p}$ is uniformly exponentially stable, we obtain for a certain constant $c^{\prime}>0$ that

$$
\begin{aligned}
\left\|t^{\alpha-1} S_{l, p}(t) f\right\|_{\infty} & \leq\left\|S_{l, p}(1)\right\|_{\mathscr{L}\left(L^{p}(\mathscr{O}), L^{\infty}(\mathscr{O})\right)}\left\|S_{l, p}(t-1)\right\|_{\mathscr{L}\left(L^{p}(\mathscr{O}), L^{\infty}(\mathscr{O})\right)}\|f\|_{p} \\
& \leq c^{\prime} e^{-\omega t}\|f\|_{p} \quad \forall t>1
\end{aligned}
$$

which is integrable on $(1, \infty)$. It thus follows that the integral in (2.1) indeed exists as a Bochner integral in $C(\overline{\mathscr{O}})$. Moreover, we have the estimate

$$
\left\|(-A)^{-\alpha} f\right\|_{\infty} \lesssim\|f\|_{p}=\left\|\left(-A_{l, p}\right) f\right\|_{\mathrm{D}\left(\left(-A_{l, p}\right)^{\alpha}\right)} .
$$

This finishes the proof.

Let us now introduce the Banach space $E$ and the operator $A$ that will be used in the abstract formulation (1.1) of equation (1.1). We set $E:=(C(\overline{\mathscr{O}}))^{r}$, endowed with the norm $\|u\|_{E}:=\sum_{l=1}^{r}\left\|u_{j}\right\|_{\infty}$. The operator $A$ will be the diagonal operator $\operatorname{diag}\left(A_{1, C}, \ldots, A_{r, C}\right)$, with domain $\mathrm{D}\left(A_{1, C}\right) \times \cdots \times \mathrm{D}\left(A_{r, C}\right)$. Then $A$ generates the strongly continuous semigroup $S_{C}:=\operatorname{diag}\left(S_{1, C}, \cdots, S_{r, C}\right)$.

To simplify notation, we will drop the index $C$ from now on and merely write $A_{1}, \ldots, A_{r}$ and $S_{1}, \ldots, S_{r}$ in what follows. 
2.2. The reaction term. Concerning the functions $f_{l}$, we make the following assumptions:

(F) For $l=1, \ldots, r$, the function $f_{l}: \overline{\mathscr{O}} \times \mathbb{R}^{k} \rightarrow \mathbb{R}$ is given as

$$
f_{l}\left(x, s_{1}, \ldots, s_{k}\right):=h_{l}\left(x, s_{l}\right)+k_{l}\left(x, s_{1}, \ldots, s_{k}\right)
$$

where the continuous function $k_{l}$ is assumed to be locally Lipschitz continuous and of linear growth in the variables $s_{1}, \ldots, s_{r}$, uniformly with respect to the first, i.e. there are constants $c_{1}, c_{2}$ and $L_{m}$ for $m \in \mathbb{N}$ such that

$$
\left|k_{l}\left(x, s_{1}, \ldots, s_{r}\right)\right| \leq c_{1}+c_{2} \sum_{j=1}^{r}\left|s_{j}\right| \quad \forall x \in \overline{\mathscr{O}}, s_{j} \in \mathbb{R}
$$

and

$$
\left|k_{l}\left(x, s_{1}, \ldots, s_{r}\right)-k_{l}\left(x, t_{1}, \cdots, t_{r}\right)\right| \leq L_{m} \sum_{j=1}^{r}\left|s_{j}-t_{j}\right| \quad \forall x \in \overline{\mathscr{O}}, s_{j}, t_{j} \in[-m, m] .
$$

The continuous function $h_{l}: \overline{\mathscr{O}} \times \mathbb{R} \rightarrow \mathbb{R}$ is assumed to be locally Lipschitz continuous in the second variable, uniformly with respect to the first. Moreover, we assume that

(1) For a certain constant $a_{l}>0$ and an integer $N_{l}$, we have

$$
-a_{l}\left(1+|s|^{2 N_{l}+1} \mathbb{1}_{\{s \geq 0\}}\right) \leq h_{l}(x, s) \leq a_{l}\left(1+|s|^{2 N_{l}+1} \mathbb{1}_{\{s \leq 0\}}\right)
$$

for all $x \in \overline{\mathscr{O}}$ and $s \in \mathbb{R}$.

(2) For certain constants $a_{1, l}, a_{2, l} \in \mathbb{R}$ and $b_{1, l}, b_{2, l}>0$ and the integer $N_{l}$ from (F1) we have

$$
a_{1, l}-b_{1, l} s^{2 N_{l}+1} \leq h_{l}(x, s) \leq a_{2, l}-b_{2, l} s^{2 N_{l}+1} .
$$

Example 2.2. Conditions (F1) and (F2) are satisfied for functions of the form

$$
h_{l}(x, s):=\sum_{j=1}^{2 N_{l}+1} \omega_{l, j}(x) s^{j}
$$

where $N_{l}$ is an integer and the coefficients $\omega_{l, j}$ belong to $C(\overline{\mathscr{O}})$ and the highest order coefficients $\omega_{l, 2 N_{l+1}}$ satisfy $\omega_{l, 2 N_{l}+1}(x) \leq-\varepsilon<0$ for some $\varepsilon>0$ and all $x \in \overline{\mathscr{O}}$. The proof of this fact can be found in Examples 4.2 and 4.5 in [14].

We now define the operators $H_{l}: C(\overline{\mathscr{O}}) \rightarrow C(\overline{\mathscr{O}})$ by $\left(H_{l}(u)\right)(x):=h_{l}(x, u(x))$ and the operators $K_{l}: E \rightarrow C(\overline{\mathscr{O}})$ by $\left(K_{l}\left(u_{1}, \ldots, u_{r}\right)\right)(x):=k_{l}\left(x, u_{1}(x), \ldots, u_{r}(x)\right)$. Finally, the map $F: E \rightarrow E$ which appears in 1.1 will be given by $F(u)=$ $\left(F_{1}(u), \cdots, F_{r}(u)\right)$, where $F_{l}(u)=H_{l}\left(u_{l}\right)+K_{l}(u)$. Obviously, the maps $F, F_{l}, H_{l}, K_{l}$ are locally Lipschitz continuous.

Conditions (F1) and (F2) imply that the maps $H_{l}$ satisfy additional dissipativity conditions which play a crucial role in proving existence of solutions of (1.1). These dissipativity conditions were first used in [7].

Recall, that in a Banach space $(B,\|\cdot\|)$, the subdifferential of the norm at $x$ is given by

$$
\partial\|x\|:=\left\{\varphi \in B^{*}:\|\varphi\|=1 \quad \text { and } \quad\langle x, \varphi\rangle=\|x\|\right\} .
$$

As a consequence of the Hahn-Banach theorem, $\partial\|x\| \neq \emptyset$ for all $x \in B$. We have

Lemma 2.3. Let $H_{l}$ be defined as above, $u, v \in C(\overline{\mathscr{O}})$ and $\varphi \in \partial\|u\|_{\infty}$. Then we have

(1) $\left\langle H_{l}(u+v), \varphi\right\rangle \leq a^{\prime}\left(1+\|v\|_{\infty}\right)^{2 N_{l}+1}$ and

(2) $\left\langle H_{l}(u+v)-H_{l}(v), \varphi\right\rangle \leq a^{\prime \prime}\left(1+\|v\|_{\infty}\right)^{2 N_{l}+1}-b^{\prime \prime}\|u\|_{\infty}^{2 N_{l}+1}$.

for suitable constants $a^{\prime}, a^{\prime \prime}, b^{\prime \prime}>0$ depending only on the constants $a_{l}$ from ( $F 1$ ) and $a_{1, l}, a_{2, l}, b_{1, l}, b_{2, l}$ from (F2). 
Proof. See [7, Section 4.3], cf. also Examples 4.2 and 4.5 in [14]

2.3. The noise term. Let us now turn to the stochastic term in equation (1.2). We shall assume:

(G) For $l=1, \cdots, r$ and $k \in \mathbb{N}$ the continuous functions $g_{l, k}: \overline{\mathscr{O}} \times \mathbb{R} \rightarrow \mathbb{R}$ have the following properties

(1) There exist $\alpha_{l}=\left(\alpha_{k}^{l}\right)_{k \in \mathbb{N}}, \beta_{l}=\left(\beta_{k}^{l}\right)_{k \in \mathbb{N}} \in \ell^{2}$ such that $\left|g_{l, k}(x, s)\right| \leq$ $\alpha_{k}^{l}+\beta_{k}^{l}|s|$ for all $x \in \overline{\mathscr{O}}$ and $s \in \mathbb{R}$.

(2) For every $m \in \mathbb{N}$ there exist continuous functions $\sigma_{l, k, m}:[0, \infty) \rightarrow$ $[0, \infty)$ such that

(a) We have $\left|g_{l, k}\left(x, s_{1}\right)-g_{l, k}\left(x, s_{2}\right)\right| \leq \sigma_{l, k, m}\left(\left|s_{1}-s_{2}\right|\right)$ for all $x \in \overline{\mathscr{O}}$ and $s_{1}, s_{2} \in[-m, m]$.

(b) For $l=1, \cdots, r$ and $m \in \mathbb{N}$, the function $\rho_{l, m}:(0, \infty) \rightarrow(0, \infty)$, defined as $\rho_{l, m}(s):=\sum_{k=1}^{\infty} \sigma_{l, k, m}(s)^{2}$ is increasing in $s$ and satisfies

$$
\int_{0+} \frac{1}{\rho_{l, m}(s)} d s=\infty .
$$

Example 2.4. Let us give an example of functions $g_{l, k}$ which satisfy $(\mathrm{G})$. For simplicity, we assume that $r=1$ and drop the subscript $l$.

Let $R: L^{2}(\mathscr{O}) \rightarrow L^{2}(\mathscr{O})$ be a Hilbert-Schmidt operator which is diagonalized by an orthonormal basis $\left(e_{k}\right)_{k \in \mathbb{N}}$, i.e. for some sequence $\lambda=\left(\lambda_{k}\right)_{k \in \mathbb{N}} \in \ell^{2}$, we have

$$
R u=\sum_{k=1}^{\infty} \lambda_{k}\left(u, e_{k}\right)_{L^{2}} e_{k}
$$

We additionally assume that $e_{k} \in C(\overline{\mathscr{O}})$ and that the sequence $\left\|\lambda_{k} e_{k}\right\|_{\infty}$ is square summable. Moreover, let $g: \mathbb{R} \rightarrow \mathbb{R}$ be of linear growth and locally Hölder continuous of order $\frac{1}{2}$, i.e. $|g(s)| \leq a+b|s|$ for certain $a, b \in \mathbb{R}$ and there exist constants $c_{m}>0$ such that $\left|g\left(s_{1}\right)-g\left(s_{2}\right)\right| \leq c_{m}\left|s_{1}-s_{2}\right|^{\frac{1}{2}}$ for all $s_{1}, s_{2} \in[-m, m]$.

Then $(\mathrm{G})$ is satisfied if we set $g_{k}(x, s):=g(s) \lambda_{k} e_{k}(x)$. Indeed, (1) holds true with $\alpha_{k}=a\left\|\lambda_{k} e_{k}\right\|_{\infty}$ and $\beta_{k}=b\left\|\lambda_{k} e_{k}\right\|_{\infty}$. For $(2)$, we choose $\sigma_{k, m}(s)=c_{m}\left\|\lambda_{k} e_{k}\right\|_{\infty} s^{\frac{1}{2}}$. Then clearly (a) is satisfied. In (b), we find $\rho_{m}(s)=s \sum_{k=1}^{\infty}\left\|\lambda_{k} e_{k}\right\|_{\infty}^{2}$, hence $\rho_{m}^{-1}$ is not integrable near 0 .

In this situation, the noise term in equation (1.2) can actually be rewritten as $g(u(t, x)) R d W(t, x)$, where $W$ is a space-time white noise, i.e. an $L^{2}(\mathscr{O})$-cylindrical Brownian motion.

Remark 2.5. If the orthonormal basis $\left(e_{k}\right)$ in example 2.4 is uniformly bounded in $C(\overline{\mathscr{O}})$, i.e. $\sup _{k}\left\|e_{k}\right\|_{\infty}<\infty$, then our assumptions reduce to $\left(\lambda_{k}\right) \in \ell^{2}$. This is for example the case if we consider the standard orthonormal basis of $L^{2}(0,2 \pi)$. If we want to consider the orthonormal basis $\left(e_{k}\right)$ which diagonalizes the operator $A_{2}$ on $L^{2}(\mathscr{O})$, then this basis consists of functions in $C(\overline{\mathscr{O}})$, as $\mathrm{D}\left(A_{s}\right) \subset C(\overline{\mathscr{O}})$, however the functions are typically not uniformly bounded in $C(\overline{\mathscr{O}})$. Typically, one can obtain a bound $\left\|e_{k}\right\|_{\infty} \lesssim k^{s}$ for a certain $s>0$ from ultracontractivity. In this case, one has to color the noise more by requiring that $\left(k^{s} \lambda_{k}\right) \in \ell^{2}$.

Let us return to our general setting and explain how the stochastic term is modeled in the abstract equation (1.1). We define $G_{l}: C(\overline{\mathscr{O}}) \rightarrow \mathscr{L}\left(\ell^{2}, C(\overline{\mathscr{O}})\right)$ by

$$
\left[G_{l}(u) h\right](x):=\sum_{k=1}^{\infty} h_{k}\left[G_{l, k}(u)\right](x),
$$

where $G_{l, k}: C(\overline{\mathscr{O}}) \rightarrow C(\overline{\mathscr{O}})$ is defined by $\left[G_{l, k}(u)\right](x):=g_{l, k}(x, u(x))$. Note that the above series converges in $C(\overline{\mathscr{O}})$, since $\left\|h_{k} g_{l, k}(\cdot, u(\cdot))\right\|_{\infty} \leq h_{k}\left(\alpha_{l, k}+\beta_{l, k}\|u\|_{\infty}\right)$ is summable. 
For the purpose of stochastic integration, we will view $G_{l}$ as a function taking values in $\mathscr{L}\left(\ell^{2}, L^{p}(\overline{\mathscr{O}})\right)$ by embedding $C(\overline{\mathscr{O}})$ into $L^{p}(\overline{\mathscr{O}})$. It turns out that $G_{l}$ even takes values in $\gamma\left(\ell^{2}, L^{p}(\mathscr{O})\right)$, the space of $\gamma$-radonifying operators from $\ell^{2}$ to $L^{p}(\mathscr{O})$.

Let us briefly recall the definition of $\gamma$-radonifying operators. Given a Hilbert space $\mathscr{H}$ and a Banach space $B$, every finite rank operators $T: \mathscr{H} \rightarrow B$ can be represented in the form

$$
T=\sum_{j=1}^{N} h_{j} \otimes x_{j}
$$

for some integer $N \geq 1$, where $\left(h_{j}\right)_{j=1}^{N}$ is an orthonormal system in $\mathscr{H}$ and $x_{1}, \ldots, x_{N} \in B$. Here, $h \otimes x$ is the rank one operator mapping $g \in \mathscr{H}$ to $(g, h)_{\mathscr{H}} x$. With $T$ represented in this form, we define

$$
\|T\|_{\gamma(\mathscr{H}, B)}:=\left(\mathbb{E}\left\|\sum_{j=1}^{N} \gamma_{j} x_{j}\right\|^{2}\right)^{\frac{1}{2}}
$$

where $\left(\gamma_{j}\right)_{j=1}^{N}$ is a sequence of independent standard normal random variables. This norm is independent of the representation of $T$ in the above form. The space $\gamma(\mathscr{H}, B)$ is the completion of the finite rank operators with respect to the norm $\|\cdot\|_{\gamma(\mathscr{H}, B)}$. The identity operator on $\mathscr{H} \otimes B$ extends to a continuous embed$\operatorname{ding} \gamma(\mathscr{H}, B) \hookrightarrow \mathscr{L}(\mathscr{H}, B)$. We may thus view $\gamma(\mathscr{H}, B)$ as a linear subspace of $\mathscr{L}(\mathscr{H}, B)$. The operators belonging to $\gamma(\mathscr{H}, B)$ are called the $\gamma$-radonifying operators from $\mathscr{H}$ to $B$. For more information about $\gamma$-radonifying operators, we refer the reader to 23 .

To see that the map $G_{l}$ from above takes values in $\gamma\left(\ell^{2}, L^{p}(\mathscr{O})\right)$, let $\left(e_{k}\right)$ denote the canonical orthonormal basis of $\ell^{2}$. Then the series

$$
\sum_{k=1}^{\infty}\left|G_{l}(u) e_{k}\right|^{2}=\sum_{k=1}^{\infty}\left|g_{l, k}(\cdot, u(\cdot))\right|^{2}
$$

converges in $L^{p}(\mathscr{O})$. Thus, by [25, Lemma 2.1], $G(u) \in \gamma\left(\ell^{2}, L^{p}(\mathscr{O})\right)$ and

$$
\|G(u)\|_{\gamma\left(\ell^{2}, L^{p}(\mathscr{O})\right)} \simeq\left\|\left(\sum_{k=1}^{\infty}\left|g_{l, k}(\cdot, u(\cdot))\right|^{2}\right)^{\frac{1}{2}}\right\|_{L^{p}(\mathscr{O})} \leq|\mathscr{O}|\left(\left\|\alpha_{l}\right\|_{\ell^{2}}+\left\|\beta_{l}\right\|_{\ell^{2}}\|u\|_{\infty}\right),
$$

proving that $G_{l}$ is of linear growth. Let us next show that $G_{l}: C(\overline{\mathscr{O}}) \rightarrow \gamma\left(\ell^{2}, L^{p}(\mathscr{O})\right)$ is continuous. To that end, let $u_{n} \rightarrow u$ in $C(\overline{\mathscr{O}})$. Employing [25, Lemma 2.1] a second time, it follows that

$$
\left\|G\left(u_{n}\right)-G(u)\right\|_{\gamma\left(\ell^{2}, L^{p}(\mathscr{O})\right)} \simeq\left\|\left(\sum_{k=1}^{\infty}\left|g_{l, k}\left(\cdot, u_{n}(\cdot)\right)-g_{l, k}(\cdot, u(\cdot))\right|^{2}\right)^{\frac{1}{2}}\right\|_{L^{p}(\mathscr{O})},
$$

which converges to 0 as $n \rightarrow \infty$ by dominated convergence.

Let us summarize the properties of $G_{l}$

Lemma 2.6. Assume $(G)$. Then the maps $G_{l}: C(\overline{\mathscr{O}}) \rightarrow \gamma\left(\ell^{2}, L^{p}(\mathscr{O})\right)$ are welldefined, of linear growth and continuous.

We now proceed to model the stochastic term in equation (1.1). The driving process $W_{H}$ is an $H$-cylindrical Wiener process for a suitable Hilbert space $H$, defined on a filtered probability space $(\Omega, \Sigma, \mathbb{F}, \mathbb{P})$, i.e. a bounded linear operator from $L^{2}([0, \infty) ; H)$ to $L^{2}(\Omega)$ with the following properties:

(1) for $f \in L^{2}([0, \infty) ; H)$, the random variable $W_{H}(f)$ is centered Gaussian.

(2) for $t>0$ and $f \in L^{2}([0, \infty) ; H)$ with support in $[0, t], W_{H}(f)$ is $\mathscr{F}_{t^{-}}$ measurable.

(3) for $t>0$ and $f \in L^{2}([0, \infty) ; H)$ with support in $[t, \infty), W_{H}(f)$ is independent of $\mathscr{F}_{t}$.

(4) for $f_{1}, f_{2} \in L^{2}([0, \infty) ; H)$ we have $\mathbb{E}\left(W_{H}\left(f_{1}\right) W_{H}\left(f_{2}\right)\right)=\left(f_{1}, f_{2}\right)_{L^{2}([0, \infty) ; H)}$. 
It is easy to see that for $h \in H$, the process $\left(W_{H}(t) h\right)_{t>0}$, defined by $W_{H}(t) h:=$ $W_{H}\left(\mathbb{1}_{(0, t]} \otimes h\right)$ is a real valued $\mathbb{F}$-Brownian motion (which is standard if $\|h\|_{H}=$ $1)$. Moreover, two such Brownian motions $\left(W_{H}(t) h_{1}\right)_{t \geq 0}$ and $\left(W_{H}(t) h_{2}\right)_{t \geq 0}$ are independent if and only $h_{1}$ and $h_{2}$ are orthogonal in $H$. We refer to 23 for a further discussion.

To model the reaction-diffusion system (1.2) in abstract form, we choose $H:=$ $\left[\ell^{2}\right]^{r}$. Denoting the canonical orthonormal basis of $\ell^{2}$ by $\left(e_{k}\right)$, we put $e_{l, k}:=$ $\left(0, \cdots, 0, e_{k}, 0, \ldots, 0\right)$, where the $e_{k}$ is at position $l$. Then $\left(e_{l, k}\right)$ is an orthonormal basis of $H$. Let $\beta_{l, k}, l=1, \ldots, r$ and $k \in \mathbb{N}$ be a family of independent realvalued Brownian motions defined on a common probability space $(\Omega, \Sigma, \mathbb{P})$. Then $W_{H}: L^{2}\left(\mathbb{R}_{+}, H\right) \rightarrow L^{2}(\Omega)$, defined by

$$
W_{H}(f):=\sum_{l=1}^{r} \sum_{k=1}^{\infty} \int_{0}^{\infty}\left(f(t), e_{l, k}\right)_{H} d \beta_{l, k}(t)
$$

is an $H$-cylindrical Wiener process, see [23. We denote by $P_{l}: H \rightarrow H$ the projection onto the $l$-th component and define $G: E \rightarrow \mathscr{L}(H, \tilde{E})$ by

$$
G(u) h:=\left(G_{1}\left(u_{1}\right) P_{1} h, \ldots, G_{r}\left(u_{r}\right) P_{r} h\right) .
$$

Later on, we will also write $H_{l}:=P_{l} H$ and define $W_{H_{l}}$ by $W_{H_{l}}(f):=W_{H}\left(P_{l} f\right)$ for $f \in L^{2}([0, \infty) ; H)$. Then $W_{H_{l}}$ is an $H_{l}$-cylindrical Wiener process.

2.4. Solution concepts. Having rewritten equation (1.2) in the abstract form (1.1), we now define what we mean by a solution of equation (1.1), thus by a solution of equation (1.2). Since the map $G$ is not locally Lipschitz continuous, we initially work with stochastically weak solutions, i.e. the probability space is part of the solution. We first consider a solution concept which is also analytically weak.

Definition 2.7. A weak solution of equation (1.1) is a tupel $\left((\Omega, \Sigma, \mathbb{P}), \mathbb{F}, W_{H}, u\right)$, where $(\Omega, \Sigma, \mathbb{P})$ is a probability space endowed with a filtration $\mathbb{F}$ which satisfies the usual conditions, $W_{H}$ is an $H$-cylindrical Wiener process with respect to $\mathbb{F}$ and $u=(u(t))_{t \geq}$ is a continuous, $\mathbb{F}$-progressive, $E$-valued process such that for all $x^{*} \in D\left(A^{*}\right)$ and $t \geq 0$ we have

$$
\left\langle u(t), x^{*}\right\rangle=\left\langle u(0), x^{*}\right\rangle+\int_{0}^{t}\left\langle u(s), A^{*} x^{*}\right\rangle+\left\langle F(u(s)), x^{*}\right\rangle d s+\int_{0}^{t} G(u(s))^{*} x^{*} d W_{H}(s) .
$$

P-almost surely.

If an initial datum $\xi \in E$ is specified, we say that $\left((\Omega, \Sigma, \mathbb{P}), \mathbb{F}, W_{H}, U\right)$ is a weak solution to the initial value problem corresponding to (1.1), if it is a weak solution of (1.1) and additionally $\mathbb{P}(U(0)=\xi)=1$.

Note that the stochastic integral in (2.2) is an integral of an $H \simeq H^{*}$-valued stochastic process with respect to a cylindrical Wiener process. Such an integral is defined as follows. If $\left(h_{k}\right)_{k \in \mathbb{N}}$ is an orthonormal Basis of $H$, put $w_{k}:=W_{H}(s) h_{k}$ which is a real valued Brownian motion. We then define

$$
\int_{0}^{t} G(u(s))^{*} x^{*} d W_{H}(s):=\sum_{k=1}^{\infty} \int_{0}^{t}\left(G(u(s))^{*} x^{*}, h_{k}\right)_{H} d w_{k}(s) .
$$

In the special situation of equation (1.2), $\mathrm{D}\left(A^{*}\right)$ can be identified with $\mathrm{D}\left(A_{1}^{*}\right) \times \cdots \times$ $\mathrm{D}\left(A_{r}^{*}\right)$ which is a subset of $\mathscr{M}(\overline{\mathscr{O}})^{r}$, the $r$-fold product of the bounded Borel measures on $\mathscr{O}$. Using the standard basis $\left(e_{l, k}\right)$ of $H=\left[\ell^{2}\right]^{r}$ and writing $\beta_{l, k}:=W_{H} e_{k l}$ as in the previous section, we have for $x^{*}=\left(\mu_{1}, \ldots, \mu_{r}\right) \in \mathrm{D}\left(A^{*}\right)$

$$
\int_{0}^{t} G(u(s))^{*} x^{*} d W_{H}(s)=\sum_{l=1}^{r} \sum_{k=1}^{\infty} \int_{\overline{\mathscr{O}}} g_{l, k}\left(x, u_{l}(s, x)\right) d \mu_{l}(x) d \beta_{l, k}(s)
$$


In particular, choosing $\mu_{l}:=R\left(\lambda, A_{l}\right)^{*} \delta_{x}$, where $\delta_{x}$ is Dirac measure in $x$ and $\mu_{j}=0$ for $j \neq l$, equation (2.2) reduces to

$$
\begin{gathered}
{\left[R\left(\lambda, A_{l}\right) u_{l}(t)\right](x)=u_{l}(0, x)+\int_{0}^{t}\left[A_{l} R\left(\lambda, A_{l}\right) u_{l}(s)\right](x)+\left[F_{l}(u(s))\right](x) d s} \\
+\sum_{l=1}^{r}\left[R\left(\lambda, A_{l}\right) G_{l}(u(s))\right](x) d W_{H_{l}}(s) .
\end{gathered}
$$

This will be used in the following section.

In order to prove existence of solutions, the concept of weak solutions is not suitable. Instead, we will use the concept of a mild solution. To define the concept of a mild solution, we have to use a Banach space valued stochastic integral. We will use the theory of stochastic integration in UMD Banach space 24]. We note that our state space $E$ is not a UMD Banach space. However, the fractional domain space $\mathrm{D}\left(\left(-A_{l, p}\right)^{\alpha}\right)$, being isomorphic to $L^{p}(\mathscr{O})$, is a UMD Banach space. We may thus perform stochastic integration in fractional domain spaces and then use Lemma 2.1 to get back into our state space. More precisely, we have

Lemma 2.8. Assume $(A)$ and $(G)$. Moreover, let $(\Omega, \Sigma, \mathbb{F}, \mathbb{P})$ be a stochastic basis on which an $H$-cylindrical Wiener process is defined. Then, if $X=\left(X_{1}, \ldots, X_{r}\right)$ is a continuous, $\mathbb{F}$-progressive E-valued process, then for every $t>0$ the process $t \mapsto S(t-s) G(X(s))$ is stochastically integrable and the stochastic integral

$$
\int_{0}^{t} S(t-s) G(X(s)) d W_{H}(s)
$$

defines an $\mathscr{F}_{t}$-measurable, E-valued random variable.

Proof. It suffices to prove the assertion for $r=1$. We will drop the index $l$ for ease of notation. Fix $\omega \in \Omega, t>0, p>\max \{2, d / 4\}$ and $\alpha \in\left(\frac{d}{2 p}, \frac{1}{2}\right)$. We claim that the map $R_{\omega}: L^{2}(0, t ; H) \rightarrow \mathrm{D}\left(\left(-A_{p}\right)^{\alpha}\right)$ given by

$$
\left\langle R_{\omega} f, x^{*}\right\rangle:=\int_{0}^{t}\left\langle S(t-s) G(X(s, \omega)), x^{*}\right\rangle d s
$$

for all $x^{*} \in \mathrm{D}\left(\left(-A_{p}\right)^{\alpha}\right)^{*}$ is $\gamma$-radonifying. Since $\mathrm{D}\left(\left(-A_{p}\right)^{\alpha}\right)$, being isomorphic to $L^{p}(\Omega)$, has type 2 , it suffices to show that $s \mapsto S(t-s) G(X(s, \omega))$ belongs to $L^{2}\left(0, t ; \gamma\left(H, \mathrm{D}\left(\left(-A_{p}\right)^{\alpha}\right)\right)\right.$, see [23, Theorem 11.6]. To that end, we have

$$
\begin{aligned}
& \|S(t-\cdot) G(X(\cdot, \omega))\|_{L^{2}\left(0, t ; \gamma\left(H, \mathrm{D}\left(\left(-A_{p}\right)^{\alpha}\right)\right)\right.}^{2} \\
= & \int_{0}^{t}\|S(t-s) G(X(s, \omega))\|_{\gamma\left(H, \mathrm{D}\left(\left(-A_{p}\right)^{\alpha}\right)\right)}^{2} d s \\
\leq & \int_{0}^{t}(t-s)^{-2 \alpha}\|G(X(s, \cdot))\|_{\gamma\left(H, \mathrm{D}\left(\left(-A_{p}\right)^{\alpha}\right)\right)}^{2} d s \\
\leq & \int_{0}^{t}(t-s)^{-2 \alpha}\left(|\mathscr{O}|\|\alpha\|_{\ell^{2}}+\|\beta\|_{\ell^{2}}\|X(\cdot, \omega)\|_{\infty}\right)^{2} d s<\infty,
\end{aligned}
$$

since $\alpha<\frac{1}{2}$. Here, we have used the estimate $\|S(t)\|_{\mathscr{L}\left(L^{p}(\mathscr{O}), \mathrm{D}\left(\left(-A_{p}\right)^{\alpha}\right)\right)} \lesssim t^{-\alpha}$ and the ideal property for $\gamma$-radonifying operators [23, Theorem 6.2] in the second line and the linear growth of $G$ from Lemma 2.6 in the third.

It now follows from [24, Lemma 2.7 and Remark 2.8], that $s \mapsto S(t-s) G(X(s, \omega))$ represents a strongly measurable map $R: \Omega \rightarrow \gamma\left(L^{2}(0, t ; H) ; \mathrm{D}\left(\left(-A_{p}\right)^{\alpha}\right)\right)$. As $s \mapsto S(t-s) G(X(s, \omega))$ is $\mathbb{F}$-progressive, it follows from [24, Theorem 5.9] that the process is stochastically integrable and the stochastic integral defines a $\mathrm{D}\left(\left(-A_{p}\right)^{\alpha}\right)$ valued random variable. By the embedding from Lemma 2.1. we are done.

We may thus define 
Definition 2.9. Assume (A), (F) and (G). A mild solution of equation (1.1) is a tupel $\left((\Omega, \Sigma, \mathbb{P}), \mathbb{F}, W_{H}, u\right)$, where $(\Omega, \Sigma, \mathbf{P})$ is a probability space endowed with a filtration $\mathbb{F}$ which satisfies the usual conditions, $W_{H}$ is an $H$-cylindrical Wiener process with respect to $\mathbb{F}$ and $u=(u(t))_{t \geq}$ is a continuous, $\mathbb{F}$-progressive, $E$-valued process such that for all $t \geq 0$ we have

$$
u(t)=S(t) u(0)+\int_{0}^{t} S(t-s) F(u(s)) d s+\int_{0}^{t} S(t-s) G(u(s)) d W_{H}(s) .
$$

P-almost surely.

If an initial datum $\xi \in E$ is specified, we say that $\left((\Omega, \Sigma, \mathbb{P}), \mathbb{F}, W_{H}, U\right)$ is a mild solution to the initial value problem corresponding to (1.1), if it is a mild solution of (1.1) and additionally $\mathbb{P}(U(0)=\xi)=1$.

By the results of [13, Section 6], the weak and the mild solutions of equation (1.1) coincide. We will thus briefly speak of solutions of equation (1.1), rather than weak (or mild) solutions.

2.5. Pathwise uniqueness and strong existence. Typically, when working with stochastically weak solutions, the appropriate uniqueness concept is that of uniqueness in law. However, we will use the following uniqueness concept:

Definition 2.10. We say that pathwise uniqueness holds for (1.1), if whenever $\left((\Omega, \Sigma, \mathbb{P}), \mathbb{F}, W_{H}, u_{j}\right)$ for $j=1,2$ are weak solutions of equation (1.1), defined on the same probability space and with respect to the same $H$-cylindrical Wiener process, satisfying $u_{1}(0)=u_{2}(0)$ a.s. we have $\mathbb{P}\left(u_{1}=u_{2}\right)=1$.

For finite dimensional stochastic differential equations, Yamada and Watanabe [29] proved that pathwise uniqueness implies uniqueness in law. Moreover, they proved that if solutions exist, then they exist strongly, i.e. given a stochastic basis $(\Omega, \Sigma, \mathbb{F}, \mathbb{P})$ on which an $H$-cylindrical Wiener process is defined, we can find a continuous, $\mathbb{F}$-progressive, $E$-valued process $u$ defined on that stochastic basis, such that $\left((\Omega, \Sigma, \mathbb{P}), \mathbb{F}, W_{H}, u\right)$ is a weak solution.

These results also generalize to stochastic equations on Banach spaces, see [13. Section 5].

We will make extensive use of strong existence of solutions in Section 5 to prove existence of solutions to stochastic reaction-diffusion systems also for unbounded reaction terms.

We should also note that given pathwise uniqueness and existence of solutions for deterministic initial values $\xi$, if follows automatically that solutions exist for random initial data $\xi: \Omega \rightarrow E$ which are $\mathscr{F}_{0}$-measurable. See 13 for a proof in the infinite dimensional case.

\section{Pathwise uniqueness}

In this section we prove

Theorem 3.1. Assume $(A),(F)$ and $(G)$. Then pathwise uniqueness holds for equation (1.2)

Proof. We may (and shall) assume that $\rho_{l, m}(t) \geq t$ for $l=1, \ldots, r$ and $m \in \mathbb{N}$, otherwise replacing $\rho_{l, m}(t)$ with $\rho_{l, m}(t)+t$.

Let us fix $l \in\{1, \cdots, r\}$ and $m \in \mathbb{N}$. Similar as in the proof of the classical Yamada-Watanabe theorem [29], we chose a decreasing sequence $a_{n} \downarrow 0$ such that $a_{0}=1$ and

$$
\int_{a_{n}}^{a_{n-1}} \frac{1}{\rho_{l, m}(t)} d t=n
$$


This is possible by assumption (G2b). Note that the sequence $\left(a_{n}\right)$, as well as the functions $\psi_{n}$ and $\varphi_{n}$ introduced next, depend on $l$ and $m$. Next we pick functions $\psi_{n} \in C_{c}^{\infty}(\mathbb{R})$ such that $\operatorname{supp} \psi_{n} \subset\left(a_{n}, a_{n-1}\right)$ and

$$
0 \leq \psi_{n}(t) \leq \frac{2}{n \rho_{l, m}(t)} \leq \frac{2}{n t} \quad \text { and } \quad \int_{a_{n}}^{a_{n-1}} \psi_{n}(t) d t=1
$$

We define

$$
\varphi_{n}(t):=\int_{0}^{|t|} \int_{0}^{s} \psi_{n}(\tau) d \tau d s .
$$

Then $\varphi_{n} \in C^{\infty}(\mathbb{R})$ with

$$
\varphi_{n}^{\prime}(t)=\operatorname{sgn}(t) \int_{0}^{|t|} \psi_{n}(s) d s \quad \text { and } \quad \varphi_{n}^{\prime \prime}(t)=\psi_{n}(|t|) .
$$

We note that

$$
|t|-a_{n-1}=\int_{0}^{|t|} \mathbb{1}_{\left(a_{n-1}, \infty\right)}(s) d s \leq \varphi_{n}(t) \leq|t|,
$$

which implies that $\varphi_{n}(t) \rightarrow|t|$, uniformly on $\mathbb{R}$. Moreover, $\varphi_{n}^{\prime}(t) t=|t| \int_{0}^{|t|} \psi_{n}(s) d s$ converges to $|t|$ pointwise.

After this preparation, we now come to the main part of the proof. Let $u_{1}:=$ $\left(u_{1}^{1}, \ldots, u_{r}^{1}\right)$ and $u_{2}=\left(u_{1}^{2}, \ldots, u_{r}^{2}\right)$ be two weak solutions of (1.2), defined on the same probability space and with respect to the same sequence of Brownian motions $\left(\beta_{l, k}\right)$. Moreover, we assume that $u_{1}(0)=u_{2}(0)$ almost surely.

For $m \in \mathbb{N}$ we define the stopping time $\tau_{m}$ by

$$
\tau_{m}:=\inf \left\{t>0:\left\|u_{1}(t)\right\|_{E} \vee\left\|u_{2}(t)\right\|_{E}>m\right\},
$$

where we set $\inf \emptyset=\infty$.

For $\lambda>0, l \in\{1, \ldots, r\}$ and $x \in \overline{\mathscr{O}}$ the vector $x^{*}=\left(\mu_{1}, \ldots, \mu_{r}\right)$, defined by $\mu_{l}^{*}:=\lambda R\left(\lambda, A_{l}\right)^{*} \delta_{x}$ and $\mu_{j}^{*}=0$ for $j \neq l$, is an element of $\mathrm{D}\left(A^{*}\right)$. Thus, since $u_{1}$ and $u_{2}$ are weak solutions with $u_{1}(0)=u_{2}(0)$ almost surely, we have, almost surely,

$$
\begin{aligned}
{\left[\lambda R\left(\lambda, A_{l}\right)\right.} & \left(u_{l}^{1}\left(t \wedge \tau_{m}\right)-u_{l}^{2}\left(t \wedge \tau_{m}\right)\right](x) \\
= & \int_{0}^{t \wedge \tau_{m}}\left[A_{l} \lambda R\left(\lambda, A_{l}\right)\left(u_{l}^{1}(s)-u_{l}^{2}(s)\right)\right](x) d s \\
& +\int_{0}^{t \wedge \tau_{m}}\left[\lambda R\left(\lambda, A_{l}\right)\left(F_{l}\left(u_{1}(s)\right)-F_{l}\left(u_{2}(s)\right)\right)\right](x) d s \\
& +\sum_{k=1}^{\infty} \int_{0}^{t} \mathbb{1}_{\left[0, \tau_{m}\right]}(s)\left[\lambda R\left(\lambda, A_{l}\right)\left(G_{l, k}\left(u_{l}^{1}(s)\right)-G_{l, k}\left(u_{l}^{2}(s)\right)\right)\right](x) d \beta_{l, k}(s)
\end{aligned}
$$

To simplify notation, we introduce some abbreviations. We write

$$
\begin{aligned}
\Delta_{\lambda} u_{l}(t) & :=\lambda R\left(\lambda, A_{l}\right)\left[u_{l}^{1}\left(t \wedge \tau_{m}\right)-u_{l}^{2}\left(t \wedge \tau_{m}\right)\right] \\
\Delta_{\lambda} F_{l}(t) & :=\lambda R\left(\lambda, A_{l}\right)\left[\left(u_{1}\left(t \wedge \tau_{m}\right)\right)-F_{l}\left(u_{2}\left(t \wedge \tau_{m}\right)\right)\right] \\
\Delta_{\lambda} G_{l, k}(t) & :=\lambda R\left(\lambda, A_{l}\right)\left[G_{l, k}\left(u_{l}^{1}\left(t \wedge \tau_{m}\right)\right)-G_{l, k}\left(u_{l}^{2}\left(t \wedge \tau_{m}\right)\right)\right] .
\end{aligned}
$$

From Itô's formula it follows that, almost surely,

$$
\begin{gathered}
\varphi_{n}\left(\Delta_{\lambda} u_{l}(t)(x)\right)=\int_{0}^{t \wedge \tau_{m}} \varphi_{n}^{\prime}\left(\Delta_{\lambda} u_{l}(s)(x)\right)\left[A_{l} \Delta_{\lambda} u_{l}(s)(x)+\Delta_{\lambda} F_{l}(s)(x)\right] d s \\
+\frac{1}{2} \int_{0}^{t \wedge \tau_{m}} \varphi_{n}^{\prime \prime}\left(\Delta_{\lambda} u_{l}(s)(x)\right) \sum_{k=1}^{\infty}\left[\Delta_{\lambda} G_{k, l}(s)(x)\right]^{2} d s+\text { a martingale. }
\end{gathered}
$$


As this is true for every $x \in \overline{\mathscr{O}}$, we may integrate over $\mathscr{O}$ and take expectations. This yields

$$
\begin{aligned}
\mathbb{E} \int_{\mathscr{O}} \varphi_{n}\left(\Delta_{\lambda} u_{l}(t)(x)\right) d x=\mathbb{E} \int_{0}^{t \wedge \tau_{m}} \int_{\mathscr{O}} \varphi_{n}^{\prime}\left(\Delta_{\lambda} u_{l}(s)(x)\right) A_{l} \Delta_{\lambda} u_{l}(s)(x) d x d s \\
+\mathbb{E} \int_{0}^{t \wedge \tau_{m}} \int_{\mathscr{O}} \varphi_{n}^{\prime}\left(\Delta_{\lambda} u_{l}(s)(x)\right) \Delta_{\lambda} F_{l}(s)(x) d x d s \\
+\frac{1}{2} \mathbb{E} \int_{0}^{t \wedge \tau_{m}} \int_{\mathscr{O}} \varphi_{n}^{\prime \prime}\left(\Delta_{\lambda} u_{l}(s)(x)\right) \sum_{k=1}^{\infty}\left[\Delta_{\lambda} G_{l, k}(s)(x)\right]^{2} d x d s \\
=: I_{1}(n, \lambda)+I_{2}(n, \lambda)+I_{3}(n, \lambda) .
\end{aligned}
$$

We proceed in several steps.

Step 1: We estimate $I_{1}(n, \lambda)$ and let $\lambda \rightarrow \infty$.

Noting that $\varphi_{n}^{\prime}\left(\Delta_{\lambda} u_{l}(s)\right) \in H^{1}(\mathscr{O})$ by the chain rule, we find that

$$
\begin{aligned}
I_{1}(n, \lambda) & =\mathbb{E} \int_{0}^{t \wedge \tau_{m}}\left(A_{l} \Delta_{\lambda} u_{l}(s), \varphi_{n}^{\prime}\left(\Delta_{\lambda} u_{l}(s)\right)\right)_{L^{2}(\mathscr{O})} d s \\
& =-\mathbb{E} \int_{0}^{t \wedge \tau_{m}} \mathfrak{a}^{l}\left[\Delta_{\lambda} u_{l}(s), \varphi_{n}^{\prime}\left(\Delta_{\lambda} u_{l}(s)\right)\right] d s .
\end{aligned}
$$

Next observe that

$$
\begin{aligned}
& \mathfrak{a}^{l}\left[\Delta_{\lambda} u_{l}(s), \varphi_{n}^{\prime}\left(\Delta_{\lambda} u_{l}(s)\right)\right] \\
= & \int_{\mathscr{O}} \sum_{i, j=1}^{d} a_{i j}^{l} \cdot\left(D_{i} \Delta_{\lambda} u_{l}(s)\right) \cdot \varphi_{n}^{\prime \prime}\left(\Delta_{\lambda} u_{l}(s)\right) \cdot\left(D_{j} \Delta_{\lambda} u_{l}(s)\right) d x \\
\geq & \int_{\mathscr{O}} \eta \varphi_{n}^{\prime \prime}\left(\Delta_{\lambda} u_{l}(s)\right) \sum_{i=1}^{d}\left|D_{i} \Delta_{\lambda} u_{l}(s)\right|^{2} d x \geq 0,
\end{aligned}
$$

since $\varphi_{n}^{\prime \prime} \geq 0$. It thus follows that $I_{1}(n, \lambda) \leq 0$.

We now abbreviate

$$
\Delta u_{l}(t):=u_{l}^{1}\left(t \wedge \tau_{m}\right)-u_{l}^{2}\left(t \wedge \tau_{m}\right), \quad \Delta F_{l}(t):=F_{l}\left(u_{1}\left(t \wedge \tau_{m}\right)\right)-F_{l}\left(u_{2}\left(t \wedge \tau_{m}\right)\right)
$$

and

$$
\Delta G_{l, k}(t):=G_{l, k}\left(u_{l}^{1}\left(t \wedge \tau_{m}\right)\right)-G_{l, k}\left(u_{l}^{2}\left(t \wedge \tau_{m}\right)\right) .
$$

For $w \in C(\overline{\mathscr{O}})$ we have that $\lambda R\left(\lambda, A_{l}\right) w$ converges to $w$ in $C(\overline{\mathscr{O}})$ as $\lambda \rightarrow \infty$. Thus, it follows that $\Delta_{\lambda} u_{l}(t) \rightarrow \Delta u_{l}(t), \Delta_{\lambda} F_{l}(t) \rightarrow \Delta F_{l}(t)$ and $\Delta_{\lambda} G_{l, k}(t) \rightarrow \Delta G_{l, k}(t)$ in $C(\overline{\mathscr{O}})$ as $\lambda \rightarrow \infty$ for every $t>0$. Inserting the estimate for $I_{1}(n, \lambda)$ into (3.1) and letting $\lambda \rightarrow \infty$ we obtain, using the continuity of $\varphi_{n}, \varphi_{n}^{\prime}$ and $\varphi_{n}^{\prime \prime}$,

$$
\begin{aligned}
\mathbb{E} \int_{\mathscr{O}} \varphi_{n}\left(\Delta u_{l}(t)(x)\right) d x \leq & \mathbb{E} \int_{0}^{t \wedge \tau_{m}} \int_{\mathscr{O}} \varphi_{n}^{\prime}\left(\Delta u_{l}(s)(x)\right) \Delta F_{l}(s)(x) d x d s \\
& +\frac{1}{2} \mathbb{E} \int_{0}^{t \wedge \tau_{m}} \int_{\mathscr{O}} \varphi_{n}^{\prime \prime}\left(\Delta u_{l}(s)(x)\right) \sum_{k=1}^{\infty}\left[\Delta G_{l, k}(s)(x)\right]^{2} d x d s \\
= & : J_{1}(n)+J_{2}(n)
\end{aligned}
$$

Step 2: We estimate $J_{1}(n)$ and $J_{2}(n)$ and let $n \rightarrow \infty$.

Since $f_{l}$ is locally Lipschitz continuous by $(\mathrm{F})$ and since $\left|\varphi_{n}^{\prime}\right| \leq 1$, it follows that, for a constant $L_{m} \geq 0$, we have

$$
J_{1}(n) \leq L_{m} \mathbb{E} \int_{0}^{t \wedge \tau_{m}} \int_{\mathscr{O}} \sum_{j=1}^{r}\left|\Delta u_{j}(s)(x)\right| d x d s \leq L_{m} \mathbb{E} \int_{0}^{t} \int_{\mathscr{O}} \sum_{j=1}^{r}\left|\Delta u_{j}(s)(x)\right| d x d s .
$$


As for $J_{2}(n)$, using $(\mathrm{G} 2)$ and the estimate $\varphi_{n}^{\prime \prime}(t)=\psi_{n}(t) \leq \frac{2}{n \rho_{l, m}(t)}$, we see that

$$
\begin{aligned}
J_{2}(n) & \leq \frac{1}{2} \mathbb{E} \int_{0}^{t \wedge \tau_{m}} \int_{\mathscr{O}} \mathbb{1}_{\mathbb{R} \backslash\{0\}}\left(\Delta u_{l}(s)(x)\right) \frac{2 \sum_{k=1}^{\infty} \sigma_{l, k, m}\left(\left|\Delta u_{l}(s)(x)\right|\right)^{2}}{n \rho_{l, m}\left(\left|\Delta u_{l}(s)(x)\right|\right)} d x d s \\
& =\frac{1}{2} \mathbb{E} \int_{0}^{t \wedge \tau_{m}} \int_{\mathscr{O}} \mathbb{1}_{\mathbb{R} \backslash\{0\}}\left(\Delta u_{l}(s)(x)\right) \frac{2 \rho_{l, m}(\Delta(s)(x)) d x}{n \rho_{l, m}\left(\left|\Delta u_{l}(s)(x)\right|\right)} d s \leq \frac{t}{n}
\end{aligned}
$$

Combining these estimates, we find that

$$
\mathbb{E} \int_{\mathscr{O}} \varphi_{n}\left(\Delta u_{l}(t)(x)\right) d x \leq L_{m} \int_{0}^{t} \int_{\mathscr{O}} \sum_{j=1}^{r}\left|\Delta u_{j}(s)(x)\right| d x d s+\frac{t}{n}
$$

Since $\varphi_{n}(t) \uparrow|t|$ as $n \rightarrow \infty$, it follows upon $n \rightarrow \infty$ that

$$
\mathbb{E} \int_{\mathscr{O}}\left|\Delta u_{l}(t, x)\right| d x \leq L_{m} \mathbb{E} \int_{0}^{t} \int_{\mathscr{O}} \sum_{j=1}^{r}\left|\Delta u_{j}(s)(x)\right| d x d s
$$

Step 3: We finish the proof.

As equation (3.2) is true for every $l=1, \ldots, r$, we find, summing up, that

$$
\mathbb{E} \int_{\mathscr{O}} \sum_{l=1}^{r}\left|\Delta u_{l}(t, x)\right| d x \leq r L_{m} \int_{0}^{t} \mathbb{E} \int_{\mathscr{O}} \sum_{l=1}^{r}|\Delta u(s)(x)| d x d s
$$

Thus, by Gronwall's Lemma,

$$
\mathbb{E} \int_{\mathscr{O}} \sum_{l=1}^{r}\left|\Delta u_{l}(t, x)\right| d x=\mathbb{E} \int_{\mathscr{O}} \sum_{l=1}^{r}\left|u_{l}^{1}\left(t \wedge \tau_{m}, x\right)-u_{l}^{2}\left(t \wedge \tau_{m}, x\right)\right| d x \equiv 0 .
$$

Since $t \wedge \tau_{m} \rightarrow t$ almost surely, upon $m \rightarrow \infty$ it follows that

$$
\mathbb{E} \int_{\mathscr{O}} \sum_{l=1}^{r}\left|u_{l}^{1}(t, x)-u_{l}^{2}(t, x)\right| d x=0
$$

for all $t \geq 0$. As solutions are continuous in $x$, it follows that $u_{1}(t)=u_{2}(t)$ almost surely, for every $t \geq 0$. Finally, by continuity of the paths, the exceptional set may be chosen independently of $t$, hence $u_{1}=u_{2}$ almost surely, i.e. pathwise uniqueness.

Remark 3.2. Note that in the proof of Theorem 3.1 neither the special structure of the functions $f_{l}$ in condition $(\mathrm{F})$, nor the linear growth assumption on the functions $g_{l, k}$ in condition (G2) was used. Thus pathwise uniqueness holds already if the functions $f_{l}$ are locally Lipschitz continuous and the functions $g_{l, k}$ satisfy (G2).

\section{Positivity}

Under additional assumptions on the nonlinearities $f_{l}$ and $g_{k, l}$, the techniques from Section 3 can also be used to prove that the solutions of equation (1.2) for positive initial data are almost surely positive. For deterministic reaction-diffusion equations, the solutions preserve positivity if and only if the reaction term is quasi positive [28].

Definition 4.1. A map $\Phi=\left(\Phi_{1}, \ldots, \Phi_{r}\right): \overline{\mathscr{O}} \times \mathbb{R}^{r} \rightarrow \mathbb{R}^{r}$ is called quasi positive if for every $l=1, \ldots, r$ and $s=\left(s_{1}, \ldots, s_{r}\right)$ with $s_{l}=0$ and $s_{j} \geq 0$ for all $j \neq l$ we have $\Phi_{l}(x, s) \geq 0$ for all $x \in \overline{\mathscr{O}}$.

In what follows, we write $s^{+}:=s \vee 0$ for the positive part of a real number $s$ and $s^{-}:=(-s) \vee 0$ for the negative part of $s$. 
Lemma 4.2. Let $\Phi: \overline{\mathscr{O}} \times \mathbb{R}^{k} \rightarrow \mathbb{R}^{k}$ be quasi positive and locally Lipschitz continuous in the sense that for $n \in \mathbb{N}$ there exists a constant $L_{n}$ such that

$$
\left|\Phi_{l}\left(x, s_{1}, \ldots, s_{r}\right)-\Phi_{l}\left(x, t_{1}, \ldots, t_{n}\right)\right| \leq L_{n} \sum_{j=1}^{r}\left|s_{j}-t_{j}\right| \quad \forall x \in \overline{\mathscr{O}}, s_{j}, t_{j} \in[-n, n] .
$$

Then for every $l \in\{1, \ldots, r\}, x \in \overline{\mathscr{O}}$ and $s_{1}, \ldots, s_{r} \in[-n, n]$ with $s_{l} \leq 0$ we have

$$
-\Phi_{l}\left(x, s_{1}, \ldots, s_{r}\right) \leq L_{n} \sum_{j=1}^{r} s_{j}^{-} .
$$

Proof. Without loss of generality, we assume that $l=1$. We have

$$
\begin{aligned}
-\Phi_{1}\left(x, s_{1}, \ldots, s_{r}\right) & =\Phi_{1}\left(x, 0, s_{2}, \ldots, s_{r}\right)-\Phi_{1}\left(x, s_{1}, \ldots, s_{n}\right)-\Phi_{1}\left(x, 0, s_{2}, \ldots, s_{r}\right) \\
& \leq L_{n}\left|0-s_{1}\right|-\Phi_{1}\left(x, 0, s_{2}, \ldots, s_{r}\right) \\
& =L_{n} s_{1}^{-}-\Phi_{1}\left(x, 0, s_{2}, \ldots, s_{r}\right) .
\end{aligned}
$$

If $\Phi_{1}\left(x, 0, s_{2}, \ldots, s_{r}\right) \geq 0$, this already yields (4.1). Otherwise, since $\Phi$ is quasi positive, there exists an index $2 \leq j_{0} \leq r$ with $s_{j_{0}}<0$. We assume without loss of generality that $j_{0}=2$. The same estimate as above shows that

$$
-\Phi_{1}\left(x, 0, s_{2}, \ldots, s_{r}\right) \leq L_{n} s_{2}^{-}-\Phi_{1}\left(x, 0,0, s_{3}, \ldots, s_{r}\right) .
$$

These arguments are now iterated. Doing this, we have proved (4.1) at some point or, after $r$ iterations, we have proved that

$$
-\Phi_{1}\left(x, s_{1}, \ldots, s_{r}\right) \leq L_{n} \sum_{j=1}^{r} s_{j}^{-}-\Phi_{1}(x, 0, \ldots, 0)
$$

which also yields (4.1), as $\Phi_{1}(x, 0, \ldots, 0) \geq 0$ by quasi positivity.

Theorem 4.3. Assume $(A),(F)$ and $(G)$. Additionally, assume that $f: \overline{\mathscr{O}} \times \mathbb{R}^{r} \rightarrow$ $\mathbb{R}^{r}$ is quasi positive and that $g_{l, k}(x, 0)=0$ for all $x \in \overline{\mathscr{O}}, l=1, \ldots, r$ and $k \in \mathbb{N}$.

If $u=\left(u_{1}, \ldots, u_{r}\right)$ is a solution of (1.2) with $u_{1}(0), \ldots, u_{r}(0) \geq 0$ almost surely, then almost surely also $u_{l}(t, x) \geq 0$ for all $t \geq 0$ and $x \in \overline{\mathscr{O}}$.

Proof. We pick the sequence $a_{n}$ and the function $\psi_{n}$ as in the proof of Theorem 3.1 and define

$$
\varphi_{n}(t):=\mathbb{1}_{(0, \infty)} \int_{0}^{t} \int_{0}^{s} \psi_{n}(\tau) d \tau d s .
$$

Then $\varphi_{n} \in C^{\infty}(\mathbb{R})$ with $\varphi_{n}(t) \uparrow t^{+}$. Moreover,

$$
\varphi_{n}^{\prime}(t)=\mathbb{1}_{(0, \infty)} \int_{0}^{t} \psi_{n}(s) d s \quad \text { and } \quad \varphi_{n}^{\prime \prime}(t)=\mathbb{1}_{(0, \infty)} \psi_{n}(t)
$$

In particular, $\varphi_{n}^{\prime}(t) \uparrow \mathbb{1}_{(0, \infty)}$ for all $t \in \mathbb{R}$.

Given a solution $u$ of (1.2) with $u_{1}(0), \ldots, u_{r}(0) \geq 0$ almost surely, we repeat the computations and estimates in the proof of Theorem 3.1 with $u_{1} \equiv 0$ and $u_{2}=: u$. Doing so, we obtain at the end of Step 1

$$
\begin{aligned}
& \mathbb{E} \int_{\mathscr{O}} \varphi_{n}\left(-u_{l}\left(t \wedge \tau_{m}, x\right)\right) \\
\leq & \mathbb{E} \int_{0}^{t \wedge \tau_{m}} \int_{\mathscr{O}} \varphi_{n}^{\prime}\left(-u_{l}\left(s \wedge \tau_{m}, x\right)\right) \cdot\left(-f_{l}\left(x, u\left(s \wedge \tau_{m}, x\right)\right) d x d s\right. \\
& +\frac{1}{2} \mathbb{E} \int_{0}^{t \wedge \tau_{m}} \int_{\mathscr{O}} \varphi_{n}^{\prime \prime}\left(-u_{l}\left(s \wedge \tau_{m}, x\right)\right) \sum_{k=1}^{\infty}\left[-g_{l, k}\left(x, u_{l}\left(s \wedge \tau_{m}, x\right)\right)\right]^{2} d x d s \\
= & : J_{1}(n)+J_{2}(n) .
\end{aligned}
$$

Note that we do not obtain an extra term for the initial datum, as $\varphi_{n}\left(-v_{l}(x)\right)=0$ almost surely. We now estimate the terms $J_{1}(n)$ and $J_{2}(n)$. 
Since $\varphi_{n}^{\prime}(t)=0$ for $t \leq 0$, the integrand in $J_{1}(n)$ vanishes, unless $u_{l}\left(s \wedge \tau_{n}, x\right)<0$. In that case, since $f$ is quasipositive and locally Lipschitz continuous, it follows from Lemma 4.2 that

$$
J_{1}(n) \leq \mathbb{E} \int_{0}^{t \wedge \tau_{m}} \int_{\mathscr{O}} \varphi_{n}^{\prime}\left(-u_{l}\left(s \wedge \tau_{m}, x\right)\right) L_{m} \sum_{j=1}^{r} u_{j}\left(s \wedge \tau_{m}, x\right)^{-} d x d s .
$$

Concerning $J_{2}(n)$, since $g_{l, k}(x, 0)=0$, we obtain

$$
\begin{aligned}
& J_{2}(n) \\
= & \frac{1}{2} \mathbb{E} \int_{0}^{t \wedge \tau_{m}} \int_{\mathscr{O}} \varphi_{n}^{\prime \prime}\left(-u_{l}\left(s \wedge \tau_{m}, x\right)\right) \sum_{k=1}^{\infty}\left[g_{l, k}(x, 0)-g_{l, k}\left(x, u_{l}\left(s \wedge \tau_{m}, x\right)\right)\right]^{2} d x d s \\
\leq & \frac{1}{2} \mathbb{E} \int_{0}^{t \wedge \tau_{m}} \int_{\mathscr{O}} \mathbb{1}_{(0, \infty)}\left(-u_{l}\left(s \wedge \tau_{m}, x\right)\right) \frac{2 \rho_{l}\left(-u_{l}\left(s \wedge \tau_{m}, x\right)\right)}{n \rho_{l}\left(-u_{l}\left(s \wedge \tau_{m}, x\right)\right)} d x d s \leq \frac{t}{n}
\end{aligned}
$$

With these estimates and $n \rightarrow \infty$, it follows that

$$
\mathbb{E} \int_{\mathscr{O}} u_{l}\left(t \wedge \tau_{m}, x\right)^{-} d x \leq L_{m} \int_{0}^{t} \mathbb{E} \int_{\mathscr{O}} \sum_{j=1}^{r} u_{j}\left(s \wedge \tau_{m}, x\right)^{-} d x d s
$$

since $\left.\varphi_{n}\left(-u_{l}\left(t \wedge \tau_{m}\right), x\right)\right) \uparrow u_{l}\left(t \wedge \tau_{m}, x\right)^{-}$.

Now the same arguments as in the proof of Theorem 3.1 show that $\sum_{l=1}^{r} u_{l}^{-}=0$ almost surely, hence, almost surely, $u_{l} \geq 0$ for all $l=1, \ldots, r$.

\section{EXISTENCE OF SOLUTIONS}

We now prove existence of solutions to equation (1.2). In the proof, we will use the pathwise uniqueness proved in Section 3. Indeed, by pathwise uniqueness, solutions exist strongly, i.e. on a given probability space and with respect to a given cylindrical Wiener process. This allows us to adopt the strategy of [14 to our situation and prove existence of solutions via approximation of the coefficients.

We will use the following estimates for deterministic and stochastic convolutions which are a consequence of the factorization technique [6].

Lemma 5.1. Assume $(A),(F)$ and $(G)$. Let $(\Omega, \Sigma, \mathbb{F}, \mathbb{P})$ be a stochastic basis on which an $H$-cylindrical Wiener process is defined. Moreover, let $u=\left(u_{1}, \ldots, u_{r}\right)$ be a continuous, $\mathbb{F}$-progressive E-valued process. Then for $p>2$ there exists a constant $C(T)$ with $C(T) \rightarrow 0$ as $T \rightarrow 0$, independent of the constants $c_{1}$ and $c_{2}$ from $(F)$ and the sequences $\alpha_{l}$ and $\beta_{l}$ from (G1) such that for the stochastic convolution process

$$
S_{l} \diamond_{l} G_{l}\left(u_{l}\right):=t \mapsto \int_{0}^{t} S_{l}(t-s) G_{l}\left(u_{l}(s) d W_{H_{l}}(s)\right.
$$

we have the estimate

$$
\mathbb{E}\left\|S_{l} \diamond_{l} G_{l}\left(u_{l}\right)\right\|_{C([0, T] ; C(\overline{\mathscr{O}}))}^{p} \leq C(T)\left(\left\|\alpha_{l}\right\|_{\ell^{2}}^{p}+\left\|\beta_{l}\right\|_{\ell^{2}}^{p} \mathbb{E}\left\|u_{l}\right\|_{C([0, T] ; C(\overline{\mathscr{O}}))}^{p}\right)
$$

and for the deterministic convolution process

$$
S_{l} * K_{l}(u):=t \mapsto \int_{0}^{t} S_{l}(t-s) K_{l}(u(s)) d s
$$

we have the estimate

$$
\mathbb{E}\left\|S_{l} * K_{l}\left(u_{l}\right)\right\|_{C([0, T] ; C(\overline{\mathscr{O}}))}^{p} \leq C(T)\left(c_{1}^{p}+c_{2}^{p} \mathbb{E}\|u\|_{E}^{p}\right)
$$

Proof. We first treat the stochastic convolution. We pick $q$ so large that $\frac{d}{2 q}<\frac{1}{2}-\frac{1}{p}$ and $\alpha \in\left(\frac{d}{2 q}, \frac{1}{2}-\frac{1}{p}\right)$. Then, for some $\varepsilon>0$ and $\beta \in\left(\alpha+\frac{1}{p}, \frac{1}{2}\right)$, we find

$$
\begin{aligned}
& \mathbb{E}\left\|S_{l} \diamond_{l} G_{l}\left(u_{l}\right)\right\|_{C([0, T] ; C(\overline{\mathscr{O}}))}^{p} \\
\lesssim & \mathbb{E}\left\|S_{l} \diamond_{l} G_{l}\left(u_{l}\right)\right\|_{C\left([0, T] ; \mathrm{D}\left(\left(-A_{l, q}\right)^{\alpha}\right)\right.}^{p}
\end{aligned}
$$




$$
\begin{aligned}
& \lesssim T^{\varepsilon p} \int_{0}^{T} \mathbb{E}\left\|s \mapsto(t-s)^{-\beta} G_{l}\left(u_{l}(s)\right)\right\|_{\gamma\left(L^{2}\left(0, t ; H_{l}\right), L^{q}(\mathscr{O})\right)}^{p} d s \\
& \lesssim T^{\varepsilon p} \int_{0}^{T} \mathbb{E}\left(\int_{0}^{t}(t-s)^{-2 \beta}\left\|G_{l}\left(u_{l}\right)\right\|_{\gamma\left(H_{l}, L^{q}(\mathscr{O})\right)}^{2} d s\right)^{\frac{p}{2}} d t \\
& \leq T^{\varepsilon p} \int_{0}^{T} \mathbb{E}\left(\int_{0}^{t}(t-s)^{-2 \beta}|\mathscr{O}|^{2}\left(\left\|\alpha_{l}\right\|_{\ell^{2}}+\left\|\beta_{l}\right\|_{\ell^{2}}\left\|u_{l}\right\|_{C([0, T ; C(\mathscr{O}))}\right)^{2} d s\right)^{\frac{p}{2}} d t \\
& \lesssim T^{\varepsilon \beta} \int_{0}^{T} \mathbb{E}|\mathscr{O}|^{p}\left(\left\|\alpha_{l}\right\|_{\ell^{2}}+\left\|\beta_{l}\right\|_{\ell^{2}}\left\|u_{l}\right\|_{C([0, T ; C(\mathscr{O}))}\right)^{p} d t \\
& \lesssim T^{\varepsilon \beta}\left(|\mathscr{O}|^{p}\left(\left\|\alpha_{l}\right\|_{\ell^{2}}^{p}+\left\|\beta_{l}\right\|_{\ell^{2}}^{p} \mathbb{E}\left\|u_{l}\right\|_{C([0, T] ; C(\mathscr{O}))}^{p}\right)\right) .
\end{aligned}
$$

Here, the first estimate follows from the embedding of Lemma 2.1, the second is [25, Proposition 4.2], the third uses that $L^{q}(\mathscr{O})$ has type 2, whence we have the embedding $L^{2}\left(0, t ; \gamma\left(H_{l}, L^{q}(\mathscr{O})\right) \hookrightarrow \gamma\left(L^{2}\left(0, t ; H_{l}\right), L^{q}(\mathscr{O})\right)\right.$. The fourth estimate follows from the linear growth of $G_{l}$, proved in Lemma 2.6. the fifth from the fact that $\sup _{t \in[0, T]} \int_{0}^{t}(t-s)^{-2 \beta} d s<\infty$ and the last one from Young's inequality. This proves the first assertion.

The (easier) proof of the section assertion is similar, using [15, Proposition 4.2.1] instead of [25, Proposition 4.2].

We first prove existence of solutions under additional boundedness assumptions on the maps $k_{l}$ and $g_{l, k}$.

Theorem 5.2. Assume $(A),(F)$ and $(G)$. Additionally assume that

(1) The functions $k_{l}$ from assumption $F$ are uniformly bounded, i.e. there exists a constant $C \geq 0$ such that $\left|k_{l}(x, s)\right| \leq C$ for all $(x, s) \in \overline{\mathscr{O}} \times \mathbb{R}^{k}$ and $l=1, \ldots, r$.

(2) For $l=1, \ldots, r$, the vector $\beta_{l}$ from assumption (G1) satisfies $\beta_{l} \equiv 0$.

Then every $\xi=\left(\xi_{1}, \ldots, \xi_{r}\right) \in E$, there exists a unique solution of equation (1.2) with initial datum $\xi$.

In the proof of Theorem 5.2, we use the following Lemma which is a reformulation of [3, Lemma 4.2], see also [14, Lemma 4.4].

Lemma 5.3. Assume that $\mathbf{T}=(T(t))_{t \geq 0}$ is a strongly continuous contraction semigroup on $C(\overline{\mathscr{O}})$ and that $H: C(\overline{\mathscr{O}}) \rightarrow C(\overline{\mathscr{O}})$ is such that for some constants $a>0$ and $N \in \mathbb{N}$ we have $\langle H(u+v), \varphi\rangle \leq a\left(1+\|v\|_{\infty}\right)^{2 N+1}$ for all $u, v \in C(\overline{\mathscr{O}})$ and $\varphi \in \partial\|u\|_{\infty}$. If for some $\tau>0, u_{0} \in C(\overline{\mathscr{O}})$ and two continuous functions $u, v:[0, \tau) \rightarrow C(\overline{\mathscr{O}})$ we have

$$
u(t)=T(t) u_{0}+\int_{0}^{t} T(t-s) F(u(s)+v(s)) d s \quad \forall t \in[0, \tau),
$$

Then

$$
\|u(t)\|_{\infty} \leq\left\|u_{0}\right\|_{\infty}+\int_{0}^{t} a\left(1+\|v(s)\|_{\infty}\right)^{2 N+1} d s \quad \forall t \in[0, \tau) .
$$

Proof of Theorem 5.2. Throughout, we fix a positive time $T>0$. It suffices to prove existence of solutions defined for times $t \in[0, T]$. Indeed, by the results of Section 3 and the Yamada-Watanabe theorem, any solution exist strongly and any two solutions agree as long as they are both defined. Thus, solutions defined on bounded time intervals can be glued together to get a solution defined for all times.

We now proceed in two steps.

Step 1 Construction of a maximal solution for equation (1.2). 
To begin with, note that by our additional assumptions (1) and (2), the operators $K_{l}$ and $G_{l}$ are uniformly bounded, i.e. there exists a constant $M \geq 0$ such that

$$
\left\|H_{l}\left(u_{1}, \ldots, u_{r}\right)\right\|_{C(\overline{\mathscr{O}})} \leq M \quad \text { and } \quad\left\|G_{l}(u)\right\|_{\gamma\left(\ell^{2}, L^{p}(\mathscr{O})\right)} \leq M
$$

for all $u_{1}, \ldots, u_{r} \in C(\overline{\mathscr{O}})$ resp. $u \in C(\overline{\mathscr{O}})$. We now also approximate the functions $H_{l}$ with uniformly bounded functions. To that end, we set

$$
h_{l}^{(n)}(x, s):= \begin{cases}h_{l}(x, s), & \text { if }|s| \leq n \\ h_{l}(x, n \cdot \operatorname{sgn} s) & \text { otherwise. }\end{cases}
$$

for $n \in \mathbb{N}$ and $l=1, \ldots, r$. We define $H_{l}^{(n)}: C(\overline{\mathscr{O}}) \rightarrow C(\overline{\mathscr{O}})$ by $\left[K_{l}^{(n)}(u)\right](x):=$ $h_{l}^{(n)}(x, u(x))$ for $u \in C(\overline{\mathscr{O}})$ and set $F_{l}^{(n)}:=K_{l}+H_{l}^{(n)}$. Then, for fixed $n \in \mathbb{N}$, the $\operatorname{map} F^{(n)}:=\left(F_{1}^{(n)}, \ldots, F_{r}^{(n)}\right): C(\overline{\mathscr{O}})^{r} \rightarrow C(\overline{\mathscr{O}})^{r}$ is uniformly bounded.

It follows from [3, Theorem 4.5] that there exists a solution $u_{n}=\left(u_{1}^{(n)}, \ldots, u_{r}^{(n)}\right)$ of the stochastic equation with coefficients $A, F^{(n)}$ and $G$ and initial datum $\xi$. By pathwise uniqueness these solutions exist strongly. Thus we may assume that the processes $u_{n}$ are defined on a common stochastic basis $(\Omega, \Sigma, \mathbb{F}, \mathbb{P})$ and that they are solutions with respect to a common $H$-Wiener process $W_{H}$. Define

$$
\rho_{n}:=\inf \left\{t \in[0, T]: \sum_{l=1}^{n}\left\|u_{l}^{(n)}(t)\right\|_{\infty}>n\right\}
$$

with the convention that $\inf \emptyset=T$.

Since $F^{(n)}(u)=F^{(n+1)}(u)$ for $u \in E$ with $\|u\|_{E} \leq n$, we can repeat the arguments in the proof of Theorem 3.1 to prove that $u_{n}(t)=u_{n+1}(t)$ for all $0 \leq t \leq \rho_{n} \wedge \rho_{n+1}$. This implies in particular that $\rho_{n} \leq \rho_{n+1}$ almost surely. Thus, putting $\rho:=\sup _{n} \rho_{n}$, we may define a maximal solution of our original problem (1.2) by $(u(t))_{t \in[0, \rho)}$, where

$$
u(t):=u_{n}(t) \quad \text { for } t \in\left[0, \rho_{n}\right] .
$$

Step 2 We show that the maximal solution $(u(t))_{t \in[0, \rho)}$ is globally defined.

We prove that for $p>2$ we have

$$
\sup _{n \in \mathbb{N}}\left\|u_{n}\right\|_{L^{p}\left(\Omega ; C\left([0, T] ; C(\overline{\mathscr{O}})^{r}\right)\right.}<\infty .
$$

By [14, Corollary 2.6] this will then imply that $\rho \equiv T$ and that $u$ is an element of $L^{p}\left(\Omega ; C\left([0, T] ; C(\overline{\mathscr{O}})^{r}\right)\right)$. It now easily follows that $u$ is a solution of our problem (1.2) on the interval $[0, T]$.

To prove (5.1), we define the stochastic processes $v_{l}^{(n)}$ and $w_{l}^{(n)}$ for $n \in \mathbb{N}$ and $l=1, \ldots r$ by

$$
v_{l}^{(n)}:=S_{l} * K_{l}\left(u_{n}\right) \text { and } w_{l}^{(n)}(t):=S_{l} \diamond_{l} G_{l}\left(u_{l}\right) .
$$

Since $H_{l}$ and $G_{l}$ are uniformly bounded it follows from Lemma 5.1 that the processes $v_{l}^{(n)}$ and $w_{l}^{(n)}$ are uniformly bounded in $L^{p}\left(\Omega ; C([0, T] ; C(\overline{\mathscr{O}}))\right.$, say by $C_{p}$.

Now put $y_{l}^{(n)}:=u_{l}^{(n)}-v_{l}^{(n)}-w_{l}^{(n)}$. Since $u_{n}$ is a mild solution of the approximate problem, it follows that

$$
y_{l}^{(n)}(t)=S_{l}(t) \xi_{l}+\int_{0}^{t} S_{l}(t-s) H_{l}^{(n)}\left(y_{l}^{(n)}(s)\right) d s+v_{l}^{(n)}(t)+w_{l}^{(n)}(t)
$$

almost surely for all $t \in[0, T]$. Note that $H_{l}^{(n)}$ satisfies assumption (F1) with the same constants as $h_{l}$. Consequently, Lemma 2.3 and Lemma 5.3 yield for $p>2$ that

$$
\mathbb{E}\left\|y_{l}^{(n)}\right\|_{C([0, T] ; C(\overline{\mathscr{O}}))}^{p}
$$




$$
\begin{aligned}
& \leq \mathbb{E} \sup _{t \in[0, T]}\left(\left\|v_{l}\right\|_{\infty}+\int_{0}^{t} a^{\prime}\left(1+\left\|v_{l}^{(n)}(s)+w_{l}^{(n)}(s)\right\|_{\infty}\right)^{2 N_{l}+1} d s\right)^{p} \\
& \lesssim \mathbb{E}\left(1+\left\|v_{l}\right\|^{p}+\left\|v_{l}^{(n)}\right\|_{C([0, T \mid ; C(\overline{\mathscr{O}}))}^{\left(2 N_{l}+1\right) p}+\left\|w_{l}^{(n)}\right\|_{C([0, T] ; C(\overline{\mathscr{O}}))}^{\left(2 N_{l+1}\right) p}\right) \\
& \leq 1+2 C_{\left(2 N_{l}+1\right) p}=: M_{l}
\end{aligned}
$$

It thus follows that

$$
\begin{aligned}
& \mathbb{E}\left\|u_{l}^{(n)}\right\|_{C([0, T] ; C(\overline{\mathscr{O}}))}^{p} \\
\lesssim & \mathbb{E}\left\|y_{l}^{(n)}\right\|_{C([0, T] ; C(\overline{\mathscr{O}}))}^{p}+\mathbb{E}\left\|v_{l}^{(n)}\right\|_{C([0, T] ; C(\overline{\mathscr{O}}))}^{p}+\mathbb{E}\left\|w_{l}^{(n)}\right\|_{C([0, T] ; C(\overline{\mathscr{O}}))}^{p} \\
\leq & M_{l}+2 C_{p} .
\end{aligned}
$$

Summing over $l=1, \ldots, r$, (5.1) is proved.

Note that assumption (F2) was not used in the proof of Theorem 5.2. That assumption is used to get rid of the additional boundedness assumption on the $G_{l}$ and $K_{l}$ in Theorem 5.2. The main tool we use is the following Lemma, which can be found in [14, Lemma 4.8].

Lemma 5.4. Assume that $\mathbf{T}=(T(t))_{t \geq 0}$ is a strongly continuous contraction semigroup on $C(\overline{\mathscr{O}})$ and that $H: C(\overline{\mathscr{O}}) \rightarrow C(\overline{\mathscr{O}})$ is such that for some constants $a, b>0$ and $N \in \mathbb{N}$ we have $\langle H(u+v)-H(v), \varphi\rangle \leq a\left(1+\|v\|_{\infty}\right)^{2 N+1}-b\|u\|_{\infty}^{2 N+1}$ for all $u, v \in C(\overline{\mathscr{O}})$ and $\varphi \in \partial\|u\|_{\infty}$. If $u, v \in C([0, \bar{T}] ; C(\overline{\mathscr{O}}))$ satisfy

$$
u(t)=\int_{0}^{t} T(t-s) H(u(s)+v(s)) d s \quad \forall t \in[0, T]
$$

then

$$
\|u\|_{C([0, T] ; C(\overline{\mathscr{O}})} \leq\left(\frac{4 a}{b}\right)^{\frac{1}{2 N+1}}\left(1+\|v\|_{C([0, T] ; C(\overline{\mathscr{O}}))}\right)
$$

Theorem 5.5. Assume $(A),(F)$ and $(G)$. Let $(\Omega, \Sigma, \mathbb{F}, \mathbb{P})$ be a stochastic basis on which an $H$-cylindrical Wiener process $W_{H}$ with respect to $\mathbb{F}$ is defined. Then for every initial datum $\xi=\left(\xi_{1}, \ldots, \xi_{r}\right) \in L^{0}\left(\Omega, \mathscr{F}_{0}, \mathbb{P} ; C(\overline{\mathscr{O}})^{r}\right)$ there exists a unique solution $u=\left(u_{1}, \ldots, u_{r}\right)$ of equation (1.2).

Proof. To proof existence of solutions we approximate the coefficients $G_{l}$ and $K_{l}$ with bounded coefficients. To that end, we define $k_{l}^{(n)}$ by $\operatorname{setting} k_{l}^{(n)}(x, s):=$ $k_{l}(x, s)$ if $\|s\|_{1} \leq n$ and $k_{l}^{(n)}(x, s):=k_{l}\left(x, n\|s\|_{1}^{-1} s\right)$ otherwise. The associated composition operator is denoted by $K_{l}^{(n)}$. The functions $g_{l}^{(n)}$ and the associated composition operator are defined analogously. Clearly, $K_{l}^{(n)}$ and $G_{l}^{(n)}$ are uniformly bounded. Moreover, they are of linear growth with constants independent of $n$.

Setting $F_{l}^{(n)}:=H_{l}+K_{l}^{(n)}$ and $F^{(n)}:=\left(F_{1}^{(n)}, \ldots, F_{r}^{(n)}\right)$, it follows from Theorem 5.2 that there exists a solution $u_{n}=\left(u_{1}^{(n)}, \ldots, u_{r}^{(n)}\right)$ of equation (1.1) with coefficients $A, F^{(n)}$ and $G^{(n)}:=\left(G_{1}^{(n)}, \ldots, G_{r}^{(n)}\right)$. Note that in Theorem 5.2 , we have proved existence of solutions only for deterministic initial data. However, as we have already remarked in Section 2.5, given pathwise uniqueness we obtain existence of solutions for arbitrary $\mathscr{F}_{0}$-measurable initial data $\xi$.

Proceeding as in the proof of Theorem 5.2, we can glue together the solutions $u_{n}$ to a maximal solution of our original problem and finish the proof of existence by proving that the approximative solutions $u_{n}$ for initial data $\xi \in L^{p}\left(\Omega, \mathscr{F}_{0}, \mathbf{P} ; E\right)$ are uniformly bounded in $L^{p}(\Omega ; C([0, T] ; E))$. This will then prove existence of solutions for $\xi \in L^{p}\left(\Omega, \mathscr{F}_{0}, \mathbf{P} ; E\right)$. The abstract results of [14] will then yield existence of solutions for $\mathscr{F}_{0}$-measurable initial data $\xi$.

To prove such a bound, we introduce the processes

$$
v_{l}^{(n)}:=S_{l} * K_{l}^{(n)}\left(u_{n}\right) \quad \text { and } \quad w_{l}^{(n)}:=S_{l} \diamond_{l} G^{(n)}\left(u_{l}^{(n)}\right) .
$$


Since the maps $K_{l}^{(n)}$ and $G_{l}^{(n)}$ are of linear growth with constants independent of $n$, it follows from Lemma 5.1 that for certain constants $d_{1}, d_{2}$ and a constant $C(T)$ which converges to zero as $T \rightarrow 0$ we have

$$
\mathbb{E}\left\|w_{l}^{(n)}\right\|_{C([0, T] ; C(\overline{\mathscr{O}}))}^{p} \leq C(T)\left(d_{1}+d_{2} \mathbb{E}\left\|u_{l}^{(n)}\right\|_{C([0, T] ; C(\overline{\mathscr{O}})}^{p}\right)
$$

and

$$
\mathbb{E}\left\|v_{l}^{(n)}\right\|_{C([0, T] ; C(\overline{\mathscr{O}}))}^{p} \leq C(T)\left(d_{1}+d_{2} \mathbb{E} \sum_{l=1}^{r}\left\|u_{l}^{(n)}\right\|_{C([0, T] ; C(\overline{\mathscr{O}})}^{p}\right) .
$$

Now we put $y_{l}^{(n)}:=u_{l}^{(n)}-v_{l}^{(n)}-w_{l}^{(n)}-S_{l}(\cdot) \xi_{l}$. Since $u_{n}$ is a mild solution of the approximate problem,

$$
y_{l}^{(n)}(t)=\int_{0}^{t} S_{l}(t-s) H_{l}\left[y_{l}^{(n)}(s)+v_{l}^{(n)}(t)+w_{l}^{(n)}(s)+S_{l}(s) \xi_{l}\right] d s .
$$

Consequently, by Lemma 5.4 .

$$
\begin{aligned}
& \mathbb{E}\left\|y_{l}^{(n)}\right\|_{C([0, T] ; C(\overline{\mathscr{O}}))}^{p} \\
\lesssim & 1+\mathbb{E}\left\|v_{l}^{(n)}+w_{l}^{(n)}+S_{l}(\cdot) \xi_{l}\right\|_{C([0, T] ; C(\overline{\mathscr{O}}))}^{p} \\
\lesssim & 1+\mathbb{E}\left\|v_{l}\right\|_{C(\overline{\mathscr{O}})}^{p}+\mathbb{E}\left\|v_{l}^{(n)}\right\|_{C([0, T] ; C(\overline{\mathscr{O}}))}^{p}+\mathbb{E}\left\|w_{l}^{(n)}\right\|_{C([0, T] ; C(\overline{\mathscr{C}}))}^{p} \\
\leq & 1+\mathbb{E}\left\|v_{l}\right\|_{C(\overline{\mathscr{O}})}^{p}+2 C(T)\left(d_{1}+d_{2} \mathbb{E} \sum_{l=1}^{r}\left\|u_{l}^{(n)}\right\|_{C([0, T] ; C(\overline{\mathscr{O}}))}^{p}\right)
\end{aligned}
$$

Combining with the estimates for $v_{l}^{(n)}$ and $w_{l}^{(n)}$ and summing over $l=1, \ldots, r$, we obtain

$$
\mathbb{E}\left\|u_{n}\right\|_{C([0, T] ; E)}^{p} \leq C_{0}+C_{1}(T) \mathbb{E}\left\|\xi_{l}\right\|_{E}^{p}+C_{2}(T) \mathbb{E}\left\|u_{n}\right\|_{C([0, T] ; E)}^{p}
$$

for certain constants $C_{0}, C_{1}(T)$ and $C_{2}(T)$, where $C_{1}(T), C_{2}(T)$ converge to 0 as $t \downarrow 0$. Thus, if we choose $T_{0}$ so small that $C_{2}\left(T_{0}\right)<1$, we obtain

$$
\mathbb{E}\left\|u_{n}\right\|_{C\left(\left[0, T_{0}\right] ; C(\overline{\mathscr{O}})^{r}\right)}^{p} \leq \frac{1}{1-C_{2}\left(T_{0}\right)}\left(C_{0}+C_{1}\left(T_{0}\right) \mathbb{E}\left\|\xi_{l}\right\|_{E}^{p}\right) .
$$

Thus we have obtained the uniform $L^{p}$-bound for small time intervals. Solving with initial datum $\tilde{\xi}:=u\left(T_{0}\right)$ and with cylindrical Wiener process $\tilde{W}_{H}(t):=W_{H}\left(t+T_{0}\right)-$ $W_{H}\left(T_{0}\right)$, we obtain solutions $\tilde{u}_{n}$. By pathwise uniqueness, $\tilde{u}_{n}(\cdot)=u_{n}\left(\cdot+T_{0}\right)$. The same arguments as above yields boundedness of $\tilde{u}_{n}$ in $L^{p}\left(\Omega ; C\left(\left[0, T_{0}\right] ; E\right)\right)$, hence boundedness of $u_{n}$ in $L^{p}\left(\Omega ; C\left(\left[0,2 T_{0}\right] ; E\right)\right)$. Inductively, we obtain boundedness in $L^{p}(\Omega ; C([0, T] ; E))$ for all $T>0$.

\section{An EXAmple}

In this section, apply our results to specific stochastic reaction diffusion system by presenting a typical example for the reaction term $f$. More precisely, we consider the situation where $r=2$, rename the variables $u:=u_{1}$ and $v=v_{2}$ and consider the function $f: \mathbb{R}^{2} \rightarrow \mathbb{R}^{2}$, defined by

$$
f_{1}(u, v):=u-u^{3}+v \quad \text { and } \quad f_{2}(u, v):=a u-b v
$$

where $a$ and $b$ are positive constants. This choice for the reaction term goes back to the fundamental work of Fitzhugh [9] and Nagumo, Arimolo and Yoshizawa 22. Reaction-diffusion equations involving this reaction term are generic excitable systems and appear frequently in applications in chemistry and biology, see e.g. [19.

This nonlinearity satisfies our assumptions (F). Indeed, setting $h_{1}(s):=s-s^{3}$ and $k_{1}(u, v):=v$, then clearly $k_{1}$ is Lipschitz continuous and of linear growth. It follows from Example 2.2 that $f_{1}$ satisfies (F1) and (F2). Moreover, can put $h_{2} \equiv 0$, which trivially satisfies $(\mathrm{F} 1)$ and $(\mathrm{F} 2)$, and $h_{2}(u, v):=u-v$. We should 
also note that the reaction term $f$ is quasi positive, as $f_{1}(0, v)=v \geq 0$ for $v \geq 0$ and $f_{2}(u, 0)=a u \geq 0$ for $u \geq 0$.

Thus, our results immediately yield the following

Theorem 6.1. Let $\mathscr{O}$ be a bounded Lipschitz domain in $\mathbb{R}^{d}, a_{1}, a_{2} \in L^{\infty}\left(\mathscr{O} ; \mathbb{R}^{d \times d}\right)$ be symmetric and uniformly elliptic. Moreover, let $R_{1}, R_{2}$ be Hilbert-Schmidt operators on $L^{2}(\mathscr{O})$ which are diagonalized by orthonormal bases $\left(e_{k}\right)$ resp. $\left(\tilde{e}_{k}\right)$ such that $e_{k}, \tilde{e}_{k} \in C(\overline{\mathscr{O}})$ for all $k$ and $\sum\left\|R_{1} e_{k}\right\|_{\infty}^{2}, \sum\left\|R_{2} e_{k}\right\|_{\infty}^{2}<\infty$. Finally, let $g_{1}, g_{2}: \mathbb{R} \rightarrow \mathbb{R}$ be locally $\frac{1}{2}$-Hölder continuous and of linear growth. Let $(\Omega, \Sigma, \mathbb{F}, \mathbb{P})$ be a stochastic basis on which two independent $L^{2}(\mathscr{O})$-cylindrical Wiener processes $W_{1}$ and $W_{2}$ are defined.

Then for every $\xi_{1}, \xi_{2} \in L^{0}\left(\Omega, \mathscr{F}_{0}, \mathbb{P} ; C(\overline{\mathscr{O}})\right)$, there exist a pathwise unique solution $(u, v)$ of the stochastic reaction diffusion system

$$
\begin{cases}d u(t) & =\left[\operatorname{div}\left(a_{1} \nabla u(t)\right)+u(t)-u(t)^{3}+v(t)\right] d t+g_{1}(u(t)) R_{1} d W_{1}(t) \\ d v(t) & =\left[\operatorname{div}\left(a_{2} \nabla v(t)\right)+a u(t)-b v(t)\right] d t+g_{2}(u(t)) R_{2} d W_{2}(t) \\ \frac{\partial u(t)}{\partial \nu_{a_{1}}}=\frac{\partial v(t)}{\partial \nu_{a_{2}}} & =0 \text { on } \partial \mathscr{O} \\ u(0) & =\xi_{1} \\ v(0) & =\xi_{2} .\end{cases}
$$

If $\xi_{1}, \xi_{2}$ are almost surely positive and $g_{1}(0)=g_{2}(0)=0$, then the solutions $u$, $v$ are almost surely positive for all times.

\section{REFERENCES}

[1] W. Arendt, Semigroups and evolution equations: functional calculus, regularity and kernel estimates, in Evolutionary equations. Vol. I, Handb. Differ. Equ., North-Holland, Amsterdam, 2004, pp. 1-85.

[2] V. BALly, I. Gyöngy, ANd É. PARdoux, White noise driven parabolic SPDEs with measurable drift, J. Funct. Anal., 120 (1994), pp. 484-510.

[3] Z. BrZeŹniaK And D. GA̧TAREK, Martingale solutions and invariant measures for stochastic evolution equations in Banach spaces, Stochastic Process. Appl., 84 (1999), pp. 187-225.

[4] Z. Brzeźniak and S. Peszat, Space-time continuous solutions to SPDE's driven by a homogeneous Wiener process, Studia Math., 137 (1999), pp. 261-299.

[5] S. CERRAI, Stochastic reaction-diffusion systems with multiplicative noise and non-Lipschitz reaction term, Probab. Theory Related Fields, 125 (2003), pp. 271-304.

[6] G. Da Prato, S. Kwapień, And J. ZabczyK, Regularity of solutions of linear stochastic equations in Hilbert spaces, Stochastics, 23 (1987), pp. 1-23.

[7] G. Da Prato And J. ZabcZYK, Nonexplosion, boundedness, and ergodicity for stochastic semilinear equations, J. Differential Equations, 98 (1992), pp. 181-195.

[8] — Stochastic equations in infinite dimensions, vol. 44 of Encyclopedia of Mathematics and its Applications, Cambridge University Press, Cambridge, 1992.

[9] R. Fitzhugh, Impulses and physiological states in theoretical models of nerve membrane, Biophys. J., 1 (1961), pp. 445-466.

[10] M. Fukushima And M. Tomisaki, Construction and decomposition of reflecting diffusions on Lipschitz domains with Hölder cusps, Probab. Theory Related Fields, 106 (1996), pp. 521557.

[11] I. Gyöngy And É. PARdoux, On quasi-linear stochastic partial differential equations, Probab. Theory Related Fields, 94 (1993), pp. 413-425.

[12] - On the regularization effect of space-time white noise on quasi-linear parabolic partial differential equations, Probab. Theory Related Fields, 97 (1993), pp. 211-229.

[13] M. C. Kunze, Martingale problems on Banach spaces. preprint. arXiv:1009.2650, 2012.

[14] M. C. Kunze and J. M. A. M. van Neerven, Continuous dependence on the coefficients and global existence for stochastic reaction diffusion equations, J. Differential Equations, 253 (2012), pp. 1036-1068.

[15] A. LunARDI, Analytic semigroups and optimal regularity in parabolic problems, Progress in Nonlinear Differential Equations and their Applications, 16, Birkhäuser Verlag, Basel, 1995.

[16] R. Manthey And T. Zausinger, Stochastic evolution equations in $L_{\rho}^{2 \nu}$, Stochastics Stochastics Rep., 66 (1999), pp. 37-85.

[17] C. Mueller, L. Mytnik, And E. Perkins, Nonuniqueness for a parabolic SPDE with $\frac{3}{4}-\varepsilon-$ Hölder diffusion coefficients. preprint. arXiv:1201.2767 2012. 
[18] C. MÜller And R. TRIBe, Stochastic p.d.e.'s arising from the long range contact and long range voter processes, Probab. Theory Related Fields, 102 (1995), pp. 519-545.

[19] J. D. Murray, Mathematical biology, vol. 19 of Biomathematics, Springer-Verlag, Berlin, second ed., 1993.

[20] L. Mytnik And E. Perkins, Pathwise uniqueness for stochastic heat equations with Hölder continuous coefficients: the white noise case, Probab. Theory Related Fields, 149 (2011), pp. 1-96.

[21] L. Mytnik, E. Perkins, And A. Sturm, On pathwise uniqueness for stochastic heat equations with non-Lipschitz coefficients, Ann. Probab., 34 (2006), pp. 1910-1959.

[22] J. Nagumo, S. Arimolo, and S. Yoshizawa, An active pulse transmission line simulating nerve axon, Proc. IRE, 50 (1962), p. 20612071.

[23] J. M. A. M. van NeERven, $\gamma$-Radonifying operators: a survey, Proceedings of the CMA, 44 (2010), pp. 1-62.

[24] J. M. A. M. van Neerven, M. C. Veraar, and L. W. Weis, Stochastic integration in UMD Banach spaces, Ann. Probab., 35 (2007), pp. 1438-1478.

[25] - Stochastic evolution equations in UMD Banach spaces, J. Funct. Anal., 255 (2008), pp. 940-993.

[26] E. M. Ouhabaz, Analysis of heat equations on domains, vol. 31 of London Mathematical Society Monographs Series, Princeton University Press, Princeton, NJ, 2005.

[27] S. Peszat, Existence and uniqueness of the solution for stochastic equations on Banach spaces, Stochastics Stochastics Rep., 55 (1995), pp. 167-193.

[28] M. PIERre, Global existence in reaction-diffusion systems with control of mass: a survey, Milan J. Math., 78 (2010), pp. 417-455.

[29] T. Yamada And S. Watanabe, On the uniqueness of solutions of stochastic differential equations., J. Math. Kyoto Univ., 11 (1971), pp. 155-167.

Graduiertenkolleg 1100, University of Ulm, 89069 Ulm, Germany

E-mail address: markus.kunze@uni-ulm.de 